\title{
Thin disk kinematics from RAVE and the solar motion
}

\author{
S. Pasetto ${ }^{1,2}$, E. K. Grebel ${ }^{2}$, T. Zwitter ${ }^{3,4}$, C. Chiosi ${ }^{5}$, G. Bertelli ${ }^{6}$, O. Bienayme ${ }^{7}$, G. Seabroke ${ }^{1}$, J. Bland-Hawthorn ${ }^{8}$, \\ C. Boeche ${ }^{2}$, B. K. Gibson ${ }^{9,10}$, G. Gilmore ${ }^{11}$, U. Munari ${ }^{6}$, J. F. Navarro ${ }^{12}$, Q. Parker ${ }^{13,14,15}$, W. Reid ${ }^{13,14}$, \\ A. Silviero ${ }^{5,16}$, and M. Steinmetz ${ }^{16}$
}

${ }^{1}$ University College London, Department of Space \& Climate Physics, Mullard Space Science Laboratory, Holmbury St. Mary, Dorking Surrey RH5 6NT, UK

e-mail: sp2@mssl.ucl.ac.uk

2 Astronomisches Rechen-Institut, Zentrum für Astronomie der Universität Heidelberg, Mönchhofstr. 12-14, 69120 Heidelberg, Germany

3 University of Ljubljana, Faculty of Mathematics and Physics, 1000 Ljubljana, Slovenia

${ }^{4}$ Center of Excellence SPACE-SI, Aškerčeva cesta 12, 1000 Ljubljana, Slovenia

5 Department of Physics and Astronomy “G. Galilei”, Padova University, Vicolo dell'Osservatorio 3, 35122 Padova, Italy

${ }^{6}$ INAF - Padova Astronomical Observatory, Vicolo dell'Osservatorio 5, 35122 Padova, Italy

7 Observatoire astronomique de Strasbourg 11 rue de l'Université, 67000 Strasbourg, France

8 Sydney Institute for Astronomy, University of Sydney, 2006 NSW, Australia

9 Jeremiah Horrocks Institute, University of Central Lancashire, Preston, PR1 2HE, UK

10 Monash Centre for Astrophysics, Monash University, 3800 Clayton, Australia

11 Institute of Astronomy, Cambridge University, Madingley Road, Cambridge CB3 OHA, UK

12 University of Victoria, Department of Physics and Astronomy, Victoria, BC V8P 5C2, Canada

13 Department of Physics and Astronomy, Macquarie University, 2109 NSW, Australia

14 Macquarie research centre in Astronomy, Astrophysics and Astrophotonics, Macquarie University, 2109 NSW, Australia

15 Australian Astronomical Observatory, PO Box 296, Epping, 2121 NSW, Australia

16 Leibniz-Institut fur Astrophysik Potsdam (AIP), An der Sternwarte 16, 14482 Potsdam, Germany

Received 23 April 2012 / Accepted 24 August 2012

\section{ABSTRACT}

\begin{abstract}
Aims. We study the Milky Way (MW) thin disk with the RAdial Velocity Experiment (RAVE) survey. We consider the thin and thick disks as different Galactic components and present a technique to statistically disentangle the two populations. Then we focus our attention on the thin disk component.

Methods. We disentangle the thin disk component from a mixture of the thin and thick disks using a data set providing radial velocities, proper motions, and photometrically determined distances.

Results. We present the trend of the velocity dispersions in the thin disk component of the MW in the radial direction above and below the Galactic plane using data from the RAVE. The selected sample is a limited subsample from the entire RAVE catalogue, roughly mapping up to $500 \mathrm{pc}$ above and below the Galactic plane, a few degrees in azimuthal direction and covering a radial extension of $2.0 \mathrm{kpc}$ around the solar position. The solar motion relative to the local standard of rest is also re-determined with the isolated thin disk component. Major results are the trend of the velocity mean and dispersion in the radial and vertical direction. In addition the azimuthal components of the solar motion relative to the local standard of rest and the velocity dispersion are discussed.
\end{abstract}

Key words. Galaxy: evolution - Galaxy: kinematics and dynamics - Galaxy: fundamental parameters

\section{Introduction}

The study of disk galaxies has played a fundamental role in the attempt to understand the intricate mechanisms that govern galaxy evolution (see, e.g., van der Kruit \& Freeman 2011, for a recent review). The complex challenge of their origin and evolution has occupied the interests of the astronomical community for a long time (e.g., Bertin 2000; Binney \& Merrifield 1998; Combes et al. 1995; Binney \& Tremaine 1987; Saslaw 1985). However, the global picture of the origin and evolution of spiral galaxies requires thorough comprehension of the structure and evolution of our own Galaxy, where many of the relevant properties and processes can be studied in detail. In this process, by mapping the Galactic disk distribution, obtaining a detailed description of our Galactic disk kinematics is of paramount importance in order to determine the structure and the evolution of the Milky Way (MW).

The first fundamental steps in this process were done by the determination of the characteristic parameters of the disk through the study of the star-count equation (e.g., Trumpler \& Weaver 1953) as in the pioneering models of Bahcall \& Soneira (1980) or Gilmore \& Reid (1983). These studies opened the way for more complete investigations by taking into account also the kinematic component of the data (e.g., Bienayme et al. 1987; Mendez et al. 1996). As a consequence of the constantly growing amount of available data and our improving knowledge of Galactic structure, the initial ideas of galaxy formation (e.g., Eggen et al. 1962; Searle \& Zinn 1978; Partridge \& Peebles 1967) have developed dramatically and merged with cosmological research becoming now either a part of a standard textbook 
picture (e.g., Binney \& Merrifield 1998; Carroll \& Ostlie 2006) or a current hot topic of research.

Continuing this long line of research the RAdial Velocity Experiment project (RAVE) was designed (see Zwitter et al. 2008; Steinmetz et al. 2006): a stellar survey that provides spectroscopic parameters for stars of the Galaxy that are mainly part of the thin disk, thick disk or halo component. The power of this survey has been repeatedly demonstrated with a series of papers focused on the principal target of the survey, i.e. the study of the Galactic structure and kinematics (e.g., Veltz et al. 2008; Casetti-Dinescu et al. 2011; Siebert et al. 2008; Hahn et al. 2011; Coşkunoğlu et al. 2011; Siebert et al. 2011; Wilson et al. 2011; Karataş \& Klement 2012), identifying stellar streams (e.g., Klement et al. 2008; Seabroke et al. 2008; Williams et al. 2011; Kiss et al. 2011) and studying the chemistry of MW components (e.g., Ruchti et al. 2010, 2011; Fulbright et al. 2010; Boeche et al. 2011; Coşkunoğlu et al. 2012).

In this paper we proceed with the general efforts devoted to understanding the properties of the Galactic disk from a kinematic point of view. Ultimately, a large spectroscopic sample of disk stars can reveal the characteristic parameters of the structure of our Galactic disk, the evolutionary history of the disk populations and the physical processes that acted on the stars in order to produce the picture that the RAVE data show us today.

Our task is to attempt to break the parameter degeneracy that results from the imprecise (or incomplete) knowledge of the observed parameters for the catalogue stars. The RAVE survey produces directly several phase space parameters. We will directly work with: the Galactic coordinates $(l, b)$, the radial velocities $v_{r} \equiv\left\langle v_{\text {hel }}, \hat{\boldsymbol{r}}\right\rangle \hat{\boldsymbol{r}}$, where the unitary vector to a generic star is a function of the directions $\hat{\boldsymbol{r}}=\hat{\boldsymbol{r}}(l, b)$. Within their errors these parameters permit us to constrain the true velocity vector once the distance is known. All these parameters come, of course, with their uncertainties. In particular, with indirect methods the distances $\boldsymbol{r}_{\text {hel }}=r_{\text {hel }} \hat{\boldsymbol{r}}$ of the stars can be derived only within a given error (e.g., Zwitter et al. 2010).

Although these quantities, if complemented by accurate distances and proper motions would provide the full phase space parameters for each star, i.e., the initial conditions for a complete $\mathrm{N}$-body integration or equivalently the boundary conditions for the Liouville equation, there are several reasons that prevent us from following this direct approach:

- the error bars of the above mentioned determinations leave too large a space of possible solutions, which prevents us from achieving an acceptable convergence of the results;

- the proximity of the stars in our sample prevents us from really following the trajectory of each star, not permitting us to evaluate the exact behaviour of more distant parts of the Galaxy.

Thus, we are in the apparently paradox situation that successful methods for external galaxies do not yield the same quality of results in our much closer Galaxy (see e.g., the results based on the full $N$-body integrations, Fux 1997, on Schwarschild methods or their more recent evolution, the Made to Measure methods e.g., by Dehnen 2009). The Galaxy description we are going to utilize here is only of statistical nature, and it is based on the collisionless Boltzmann approximation to describe the Galaxy as a set of two components (we are considering only thin and thick disk stars in our description). This will prevent us from easily deriving any consideration of dynamical nature, and we will limit ourselves to a simpler kinematic description. In particular, here we present a statistically determined description of the thin disk component alone that can be achieved, within reasonable errors, in the solar neighbourhood.

Nonetheless, the problem of extending the kinematic parameters estimated here for the solar neighbourhood to the entire disk can be justified based on symmetry considerations and on the comparison with external spiral galaxies. If we limit ourselves to a kinematic description, i.e., a steady state description for a one-component disk (namely the thin disk), then the Jeans theorem (e.g., Binney \& Tremaine 1987) authorizes us to look at the solar vicinity as a paradigm of the Galactic disk kinematics (e.g., May \& Binney 1986) as long as we do not attempt too detailed a description, (e.g., Quillen et al. 2011). We will extensively discuss the limitation of our approach in later sections of our paper.

The structure of this paper is the following: in Sect. 2, we review the hypotheses of the methodology adopted and the data selection criteria. In the Sect. 3 we present the results and in Sect. 4 our conclusions.

\section{Methodology adopted}

\subsection{Target}

In this paper, our target is to present the trend of the first two moments of the velocity distribution (the mean and the central moment of order 2 ) for the thin disk component along the meridional plane $(O, R, z)$ of the MW. Using a methodology recently developed in Pasetto et al. (2012, hereafter Paper I) we are able to disentangle the thin disk and thick disk components from the mixture of the two disk populations near the sun. Then, with the help of proper motions and distances photometrically determined by Zwitter et al. (2010) we can follow the trend of the thin disk velocity ellipsoid described in cylindrical coordinates $(O, R, \varphi, z)$.

\subsection{Data selection}

We review here the data selection, while the methodology is fully explained in Paper I. We used an internal release of the RAVE data catalogue equipped with photometrically determined distances (Zwitter et al. 2010), radial velocities and proper motions. This provides us with a full phase space description for about 210000 stars consisting of a mixture of thin and thick disk and halo stars.

In order to limit our sample only to the thin and thick disk components, we select from the RAVE catalogue all the stars with $\log _{10} g>3.5$ (with $g$ denoting the surface gravity of the star), the near-infrared colours from the Two Micron All Sky Survey (2MASS, Skrutskie et al. 2006, $J-K \in[0.3,1.1]$ mag in the $J$ and $K$ band (see Steinmetz et al. 2006; and Zwitter et al. 2008, for details), the latitude of the sample $|b|>10 \mathrm{deg}$, $[\mathrm{Fe} / \mathrm{H}]>-1$ (from Zwitter et al. 2008 in order to minimize any halo contamination), $\left\|\boldsymbol{v}_{\text {hel }}\right\|<150 \mathrm{~km} \mathrm{~s}^{-1}$ as absolute value of the true velocity vector and $S / N>20$ for the signal-to-noise ratio of the spectra. Finally, we cut the sample with the kinematical condition investigated in Paper I: $\Delta v_{r}^{\mathrm{RAVE}}\left(l_{i}, b_{i}\right)>2.0 \delta v_{r}^{\mathrm{G}}\left(l_{i}, b_{i}\right)$ (Eq. (6) in Paper I) where $\delta v_{r}^{\mathrm{G}}\left(l_{i}, b_{i}\right)$ is the difference between a maximum value $\max \left(v_{r}^{\mathrm{G}}\right)$ and a minimum value $\min \left(v_{r}^{\mathrm{G}}\right)$ of the Galactic component of the radial velocity in the direction of the star $s_{i}$ where $r \in[0,1] \mathrm{kpc}$. This condition has been investigated in Paper I in order to reduce the indetermination of the kinematical parameters for the most distant stars and the large scale effects along the selection process. An isocontour plot of this inequality based on the assumed Galactic gravitational potential 


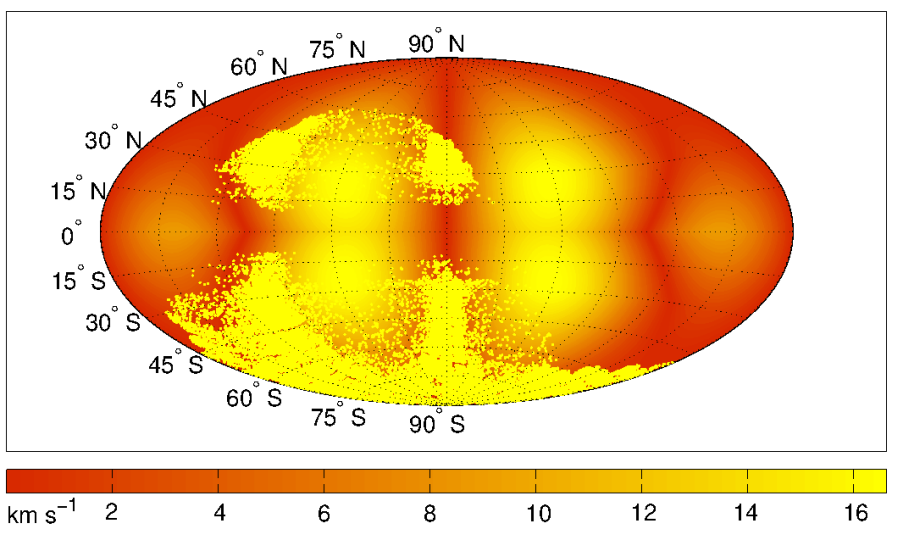

Fig. 1. Celestial sphere distribution in an Aitoff projection of the selected stellar sample (yellow dots) as outlined in the text. The background density map is explained in the text.

field (e.g., Bertelli et al. 2003; Vallenari et al. 2006) is given in the background contour plot in Fig. 1 and can be directly compared with Fig. 1 of Paper I. As we can see from this contour plot, the highest $\delta v_{r}^{\mathrm{G}}\left(l_{i}, b_{i}\right)$ (yellowish zones) occur at low latitude and the smallest in the red zones. For $b=0, \delta v_{r}^{\mathrm{G}}\left(l_{i}, b_{i}\right)$ is the terminal velocity relation and we see that the largest induced error is of the order of $16 \mathrm{~km} \mathrm{~s}^{-1}$ at a distance of $1 \mathrm{kpc}$ and at low Galactic latitudes not covered by the RAVE survey. Here we assume that the true velocity vector is defined in a suitable reference system for the velocity space $(O, U, V, W)$ aligned with the configuration reference system at the solar position. The $U$ direction points to the Galactic coordinates $(l, b)=(180,0)$ deg, $V$ runs through $(l, b)=(90,0)$ deg and $W$ points to the north Galactic pole (NGP).

This data selection leaves us with a sample of 38805 dwarf stars that map the solar neighbourhood's stellar distribution avoiding the influence from the small halo component (see Fig. 1).

\subsection{Disentangling technique}

In the classical kinematic description, a single collisionless stellar population satisfies the collisionless Boltzmann equation, i.e., a linear and homogeneous partial differential equation in the phase space distribution function (DF), $f$ (once the total potential $\Phi_{\text {tot }}$ of the whole Galaxy is assumed). Since this equation is linear in $f$, the whole system can be described as a mixture of populations, e.g., $f=\sum_{i} f_{i}$. In Paper I (to which we refer the reader for further details) a methodology based on the singular value decomposition solution for overdetermined systems was outlined in order to statistically derive the first moments of a DF $f_{\text {mix }}=f_{\text {thin }}+f_{\text {thick }}$ for a stellar mixture of thin and thick disks. Then, we statistically disentangled the thick disk population alone, assuming a generalized ellipsoidal DF, $f_{\text {thick }}$, in a modification of the original work of Cubarsi \& Alcobé (2004).

The selection procedure works only on the radial velocity. At every iteration, say $j$, by randomly choosing a radial velocity value within its error bar, say $v_{r}^{[j]}\left(s_{i}\right)$ for each star, $s_{i} \in P^{[j]}$, i.e. after selection criteria in Sect. 2.2, we are left with a selected RAVE sample of stars $P^{[j]}$ within their associated errors:

$P^{[j]} \equiv\left\{s_{i} \mid v_{r}^{[j]}\left(s_{i}\right) \in\left[v_{r}\left(s_{i}\right)-\Delta v_{r}\left(s_{i}\right), v_{r}\left(s_{i}\right)+\Delta v_{r}\left(s_{i}\right)\right]\right\}$.

The procedure then isolates the thick disk component by randomly excluding stars in order to optimize a statistical weight parameter $q$ described in Paper I (see also Cubarsi \& Alcobé 2004). Once the population that maximizes $q$ within the machine error precision (namely $q=\infty$ ) has been isolated, we are naturally left with two samples. The sample $S^{[j]}$, with $S^{[j]} \subset P^{[j]} \forall j$, describing the thick disk and the sample $P^{[j]} \backslash S^{[j]}$ or $\left[\neg S^{[j]}\right]$ (read "not $P^{[j]}$ ") describing the thin disk, is defined as:

$$
T^{[j]} \equiv P^{[j]} \backslash S^{[j]}=\left\{s_{i} \mid s_{i} \in P^{[j]} \wedge s_{i} \notin S^{[j]}\right\} \forall j
$$

thus once the thick disk sequence of stars for the iteration $j$ is removed all the other stars are considered to be thin disk stars. We explicitly state that:

- we do not limit the determination of the errors to a preselected sample of stars. For instance, a generic star $s_{i}$ of $P^{[j]}$ can belong to the sample $S^{[j]}$ for the iteration number $j$ and to the sample $T^{[j]}$ for the iteration number $j+1$. The indices $j$ and $i$ are not correlated;

- the thin disk selection is based only on the radial velocities and their errors once the sample $P$ has been chosen. This makes use of the good quality errors as determined by the RAVE survey in Eq. (1).

We emphasize that the first point is fundamental: we are not assigning any probability weight to a single star in the sense of being a thin disk or thick disk star, even though we know its full phase space description. This assigns much more generality to our method and prevents us from biasing the results.

A schematic view of how the method works is sketched in Fig. 2 where for a generic sample $P^{[j]}$, the direction to each star, $\hat{\boldsymbol{r}}$, is represented by grey arrows (solid for the thin disk stars and dashed for the thick disk). The true, unknown velocity vectors for the thick disk stars $\boldsymbol{v}_{\text {hel }}^{\text {thick }}$, drawn from the DF $f_{\text {thick }}$, are represented in light red. The sketch shows the thin disk star velocity vectors, $\boldsymbol{v}_{\text {hel }}^{\text {thin }}$, with dark red arrows that are slightly greater closer to the Galactic centre (GC) and smaller in the radial outward direction for $R>R_{\odot}$, with $R_{\odot}$ being the solar position. This is an expected trend for the thin disk velocity ellipsoid which is supposed to have larger ellipsoidal axes in the inner zones of the Galaxy and smaller ones outside (e.g., Binney \& Merrifield 1998). The radial projection of each velocity vector is shown with red arrows: thinner for the thin disk and thicker for the thick disk. We used in the selection process only the radial velocity (red arrows) $\boldsymbol{v}_{\|}=\left\langle\boldsymbol{v}_{\text {hel }}, \hat{\boldsymbol{r}}\right\rangle \hat{\boldsymbol{r}}$ provided by RAVE, and thanks to the different velocity dispersion of the thin and thick disk components (graphically realized with longer and shorter red arrows) we managed to disentangle the thick disk component as in Paper I. Once the thick disk stars have been selected (dashed grey arrows) we call all the other stars "thin disk stars" (the solid grey arrows). We determine their true velocity vectors (dark red arrows) by making use of the proper motion values tabulated in the RAVE internal data release and calibrated with photometrically determined distances from Zwitter et al. (2010). In addition we use the matrix transformation of Johnson \& Soderblom (1987) corrected to J2000 as in Pasetto et al. (2003) in order to derive the velocity dispersion tensor for the thin disk alone.

\section{Results}

We present now the analysis of the averaged results for a large set of statistical realizations of the sample $T^{[j]}$ for the single thin disk component (typically $j=1, \ldots, N$ with $N>1024$ ) as outlined in the previous section. With the concept of a single component we mean each part of the system that is characterized by a specific property, i.e. the chemical elements $\mathbb{C}$, the age $t$, 
Table 1. Trend of the mean radial velocity $\bar{v}_{R}$ expressed in $\left[\mathrm{km} \mathrm{s}^{-1}\right]$ and the distance from the Galactic plane bins are in [kpc].

\begin{tabular}{lcccccc}
\hline \hline $\bar{v}_{R}(R, z)$ & $<-0.6$ & ]$-0.5,-0.3]$ & ]$-0.3,-0.1]$ & ]$-0.1,0.1]$ & ] $0.1,0.3]$ & ] $0.3,0.5]$ \\
\hline 7.6 & & $21.7 \pm 50.0$ & & & $63.1 \pm 0.0$ & \\
7.8 & $35.3 \pm 67.2$ & $14.2 \pm 12.1$ & $12.8 \pm 28.0$ & & $8.2 \pm 21.3$ & $17.5 \pm 20.1$ \\
8.0 & $3.7 \pm 6.6$ & $16.4 \pm 7.5$ & $9.1 \pm 4.3$ & & $13.2 \pm 9.3$ & $14.4 \pm 9.6$ \\
8.2 & $20.4 \pm 24.5$ & $14.9 \pm 4.4$ & $9.9 \pm 2.6$ & $10.0 \pm 11.9$ & $13.3 \pm 4.5$ & $16.4 \pm 8.2$ \\
8.4 & $19.2 \pm 18.7$ & $11.4 \pm 3.1$ & $10.9 \pm 2.7$ & $10.9 \pm 1.0$ & $13.4 \pm 2.6$ & $12.5 \pm 4.9$ \\
8.6 & & $10.4 \pm 3.1$ & $10.2 \pm 1.6$ & $10.1 \pm 2.6$ & $11.5 \pm 2.4$ & $5.7 \pm 2.6$ \\
8.8 & $6.5 \pm 11.6$ & $6.1 \pm 3.1$ & $7.4 \pm 2.2$ & $17.2 \pm 45.3$ & $6.4 \pm 4.0$ & $8.0 \pm 6.5$ \\
9.0 & $-6.2 \pm 31.7$ & $6.7 \pm 3.8$ & $10.3 \pm 10.9$ & & $9.4 \pm 26.0$ & $4.1 \pm 3.5$ \\
9.2 & & $-3.8 \pm 10.6$ & $12.9 \pm 0.0$ & & & \\
9.2 & & $22.4 \pm 0.0$ & & & & \\
\hline
\end{tabular}

Notes. Empty slots indicate parameter ranges with too few stars.

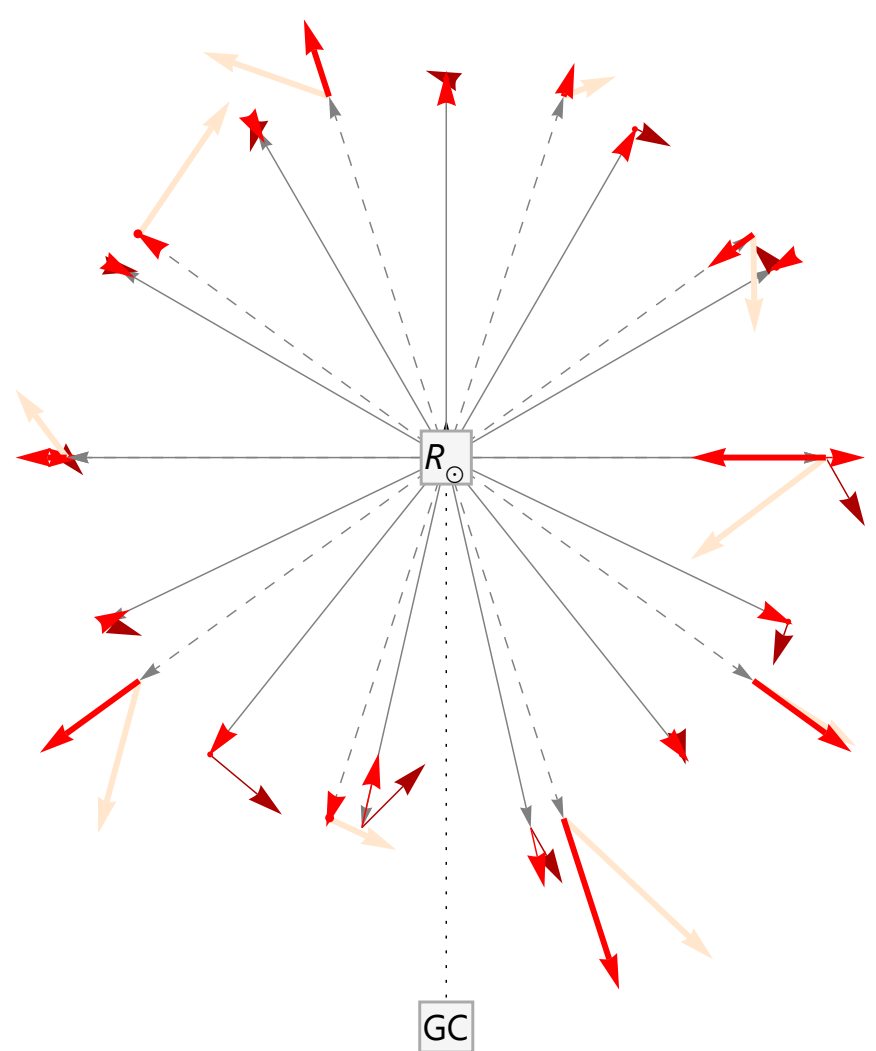

Fig. 2. Sketch of the geometry exploited by the method. The observer is located at the Sun's position $R_{\odot}$ at the centre. The Galactic centre (GC) is at the bottom of the figure. The directions on the celestial sphere to each star $s_{i}$ are indicated with dashed arrows for thick disk stars (the sample $S^{[j]}$ ) and solid arrows for the thin disk stars (the sample $T^{[j]}$ ). The red thick arrows represent the radial velocities of the thick disk, whose corresponding unknown true velocity vector is in light red. The thin disk radial velocities are represented by the thin red arrows, and the corresponding unknown dark red arrows represent the true velocity vectors. Note that the dark red arrows are generally longer in the GC direction and smaller in the anti-Galactic centre direction as expected from the velocity dispersion trend.

the mass-to-light ratio $M / L$ in some specific band, the kinematic parameters, the scale length, etc. If we collectively call $\Xi$ the sample of the physical properties that we are not interested in, its cardinality (i.e. the number of its elements) can be very high, or equal to the cardinality of the continuum. We will average the DF over all the properties we do not wish to study so that we will refer to the thin (or thick disk) DF simply as $f_{\text {thin }} \propto \int f_{\text {thin }} \mathrm{d} \Xi$. Since the mapping of the meridional plane kinematics is our principal goal, we will limit ourselves to the kinematic description of the thin disk DF (through only its first and second velocity moment). Thus once the sample of stars $T^{[j]}$ is selected, we are left with the proper motion and their errors, radial velocities and their errors, distances and their errors $\forall s_{i} \in T^{[j]}$ (and the sky directions considered without errors).

\subsection{Data binning}

Once the thin disk velocity vectors $\boldsymbol{v}_{\text {hel }}$ have been found, we proceed by binning the sample in the meridional plane. We are not binning in the $\phi$ direction in order to exploit the axisymmetric assumption we made when the selection procedure was developed as explained in Paper I, i.e. we are summing up all the $\phi_{s_{i}}$ for each star $s_{i}$ and the Sun at the azimuthal position $\phi_{\odot}=0$ in a Galactocentric cylindrical reference system. The choice of the bin size does not affect the results we are presenting once a sufficiently large bin (say, $\geqslant 100 \mathrm{pc}$ ) is adopted since we are interested in the general trend of a smooth function of the independent variables $R$ and $z$ (and not in the description of the granularity of the DF). Different statistical approaches can be shown to give the same physical results, e.g., the statistical estimators in Vio et al. (1994).

\subsection{The mean motion}

In Tables 1-3 the velocity components of the mean velocity vector are listed along with their errors for a selected sample of bins. A common trend is present in the values of the tables, although it is also evident that the large error bars prevent us from obtaining a stable fitting function. This means that although the average values for $\bar{v}_{R}, \bar{v}_{\phi}$ and $\bar{v}_{z}$ show a smooth trend, we will not be in the position to compute their derivatives $\partial_{R} \bar{v}_{i}$ or $\partial_{z} \bar{v}_{i}$ which remain a primary target of forthcoming surveys such as Gaia. In general the tables show a lack of data in the Galactic centre and anticentre directions as a consequence of the selection procedure outlined in Sect. 2.2 and the Galactic latitude selection cuts $(|b|>10 \mathrm{deg})$. Nevertheless, when possible, we retain values as e.g., $\bar{v}_{R}=21.7 \pm 50.0 \mathrm{~km} \mathrm{~s}^{-1}$ in Table 1 for the distance range $z \in]-0.5,-0.3] \mathrm{kpc}$ (and similar in Tables 2 and 3), because their mean values are in agreement with the general trend of the analysed property, e.g., $\bar{v}_{R}$, expressed by the neighbouring bins in the tables. 
Table 2. Trend of the mean circular velocity $\bar{v}_{\phi}$ expressed in $\left[\mathrm{km} \mathrm{s}^{-1}\right]$ and the distance from the Galactic plane bins are in [kpc].

\begin{tabular}{lcccccc}
\hline \hline $\bar{v}_{\phi}(R, z)$ & $<-0.6$ & ]$-0.5,-0.3]$ & ]$-0.3,-0.1]$ & ]$-0.1,0.1]$ & ] $0.1,0.3]$ & ] $0.3,0.5]$ \\
\hline 7.6 & & $6.6 \pm 17.1$ & & & & \\
7.8 & $-43.1 \pm 78.5$ & $-12.5 \pm 8.3$ & $12.5 \pm 26.2$ & & $2.7 \pm 7.7$ & $2.2 \pm 3.6$ \\
8.0 & $-47.2 \pm 61.9$ & $-10.8 \pm 3.9$ & $2.5 \pm 1.6$ & & $5.8 \pm 4.6$ & $-8.0 \pm 3.0$ \\
8.2 & $-42.6 \pm 45.9$ & $-4.5 \pm 1.2$ & $6.0 \pm 1.6$ & $7.8 \pm 8.8$ & $6.1 \pm 2.9$ & $-8.6 \pm 2.8$ \\
8.4 & $-36.6 \pm 41.0$ & & $8.3 \pm 1.5$ & $11.3 \pm 1.5$ & $7.2 \pm 0.9$ & $-5.7 \pm 2.0$ \\
8.6 & $-42.6 \pm 58.0$ & $-2.7 \pm 0.5$ & $9.9 \pm 2.6$ & $13.1 \pm 2.9$ & $9.2 \pm 2.2$ & $-7.8 \pm 2.9$ \\
8.8 & $-44.7 \pm 140.4$ & $-3.0 \pm 0.6$ & $9.5 \pm 4.3$ & $9.2 \pm 29.6$ & $7.2 \pm 5.8$ & $-11.2 \pm 8.4$ \\
9.0 & $-29.6 \pm 103.9$ & $-5.8 \pm 4.1$ & $5.0 \pm 7.7$ & & $5.5 \pm 20.9$ & $-11.7 \pm 24.9$ \\
9.2 & & $-2.6 \pm 4.3$ & & & & \\
\hline
\end{tabular}

Notes. Empty slots indicate parameter ranges with too few stars. Refer to the text for the limitations of our approach along this direction.

Table 3. Trend of the mean vertical velocity $\bar{v}_{z}$ expressed in $\left[\mathrm{km} \mathrm{s}^{-1}\right]$ and the distance bins from the Galactic plane are in [kpc].

\begin{tabular}{lcccccc}
\hline \hline $\bar{v}_{z}(R . z)$ & $<-0.6$ & ]$-0.5 .-0.3]$ & ]$-0.3 .-0.1]$ & ]$-0.1 .0 .1]$ & ]0.1.0.3] & ]0.3.0.5] \\
\hline 7.6 & & $18.8 \pm 45.2$ & & & & \\
7.8 & & $15.6 \pm 13.1$ & $15.1 \pm 32.7$ & & $22.0 \pm 56.6$ & $10.8 \pm 13.3$ \\
8.0 & $14.9 \pm 20.3$ & $13.5 \pm 7.3$ & $10.3 \pm 6.5$ & & $6.8 \pm 4.5$ & $10.5 \pm 5.8$ \\
8.2 & $18.5 \pm 22.1$ & $10.3 \pm 4.2$ & $9.0 \pm 2.0$ & $5.4 \pm 7.9$ & $8.8 \pm 2.3$ & $10.0 \pm 4.1$ \\
8.4 & $13.4 \pm 15.5$ & $10.7 \pm 4.3$ & $8.1 \pm 1.2$ & $7.2 \pm 1.3$ & $9.2 \pm 2.7$ & $13.4 \pm 7.2$ \\
8.6 & $16.2 \pm 23.6$ & $9.9 \pm 3.7$ & $9.4 \pm 2.0$ & $8.1 \pm 3.1$ & $10.3 \pm 3.7$ & $16.2 \pm 8.8$ \\
8.8 & $5.1 \pm 16.5$ & $8.2 \pm 3.6$ & $10.8 \pm 4.3$ & $10.0 \pm 33.3$ & $12.5 \pm 11.8$ & $17.8 \pm 18.0$ \\
9.0 & $21.3 \pm 75.9$ & $9.6 \pm 9.4$ & $12.3 \pm 19.5$ & & $5.2 \pm 20.7$ & $11.2 \pm 26.2$ \\
9.2 & & $25.8 \pm 47.1$ & & & & \\
\hline
\end{tabular}

Notes. Empty slots indicate parameter ranges with too few stars.

\subsubsection{Mean radial motion}

The trend of the mean radial motion $\bar{v}_{R}$ is presented in Table 1 . The column describing the vertical range $z \in]-0.1,0.1] \mathrm{kpc}$ contains the closest and our best-determined values in the solar neighbourhood. Assuming the location of the Sun to be in the range $R \in] 8.4,8.6] \mathrm{kpc}$ we recover a value of $\bar{v}_{R}=$ $10.9 \pm 1.0 \mathrm{~km} \mathrm{~s}^{-1}$, in general agreement with the recent independent determination (e.g., Aumer \& Binney 2009) but with a larger error bar due to the statistical approach adopted. This value is in good agreement also with our recent determination deduced from the thick disk component alone in Paper I. Also, the value of $\bar{v}_{R}$ seems to be robust against possible large scale effect corrections (see also, Schönrich 2012). Table 1 provides us with a consistent value for the LSR radial motion not only within the closest radial bin around the solar location, but also in its extended neighbourhood. Table 1 shows no net global motion within the error bars, i.e., the values show no net global radial motion of the stars with respect to the motion of the Sun. This is an indication of the absence of a net radial motion trend with respect to the solar motion and is in line with results already obtained much closer inside and further outside of our range of distances in the milestone paper of Lewis \& Freeman (1989). This means that for a mixed set of stellar populations, the assumption of a zero net expansion is quite reasonable within the error bars even if a small outward trend can be evinced from the plot. On the plane $z=0$, the radial motion of the stars at the solar position remains essentially unchanged from $-0.5 \mathrm{kpc}$ to $+0.5 \mathrm{kpc}$. Similar considerations hold along all radial extensions of the RAVE data sample within or outside the solar radial position for above and below the plane respectively.

\subsubsection{Mean circular motion}

The values for the mean circular motion $\bar{v}_{\phi}$, are presented in Table 2. The solar neighbourhood value for this component is
$v_{V, \odot}=11.3 \pm 1.5 \mathrm{~km} \mathrm{~s}^{-1}$, which is slightly higher than the classical literature values (e.g., Binney \& Merrifield 1998) but agrees with recent values such as in Piskunov et al. (2006) and Schönrich et al. (2010). Nevertheless, this value is slightly more difficult to estimate because of the superposition of several effects: the Galactic rotation, the peculiar velocity of the Sun relative to the local standard of rest at the solar position (LSR), the distribution of velocities of the sample of stars, and finally the presence of the thick disk stars. Here we have statistically excluded the thick disk's kinematic contribution to this value. The relation between the other contributors, for a single component thin disk model, can be approximated with the help of the Jeans equation as

$$
\begin{aligned}
\left|\overline{\boldsymbol{v}}_{c}(R, z)\right|= & {\left[V_{\mathrm{LSR}}^{2}-\frac{\partial \ln \rho}{\partial \ln R}\left(\sigma_{\mathrm{RR}}^{2}+\sigma_{\mathrm{Rz}}^{2}\right)+\left(\sigma_{\mathrm{RR}}^{2}+\sigma_{\phi \phi}^{2}\right)\right.} \\
& \left.+R\left(\frac{\partial \sigma_{R R}^{2}}{\partial R}+\frac{\partial \sigma_{R z}^{2}}{\partial z}+\frac{\partial \Phi_{\mathrm{tot}}}{\partial R}\right)\right]^{1 / 2}
\end{aligned}
$$

with $V_{\mathrm{LSR}}^{2}(R)$ as the velocity of the circular orbit at the radius $R, \rho$ as the stellar population density profile and $\sigma_{i j}$ the velocity dispersion tensor elements introduced below. This equation shows how part of the kinetic energy of any disk population goes into rotation and part in the velocity dispersion, which is necessary to stabilize the disk by achieving a suitable $Q$ parameter (after Toomre 1964). Nevertheless, there are a few important reasons why we cannot easily exploit the previous equation in order to disentangle the different contributions to the mean circular velocity:

1. This result is an expected shortcoming of the methodology adopted to disentangle the thin and thick disk components. As already explained in Paper I, although the collisionless Boltzmann equation is linear in the phase space distribution function, the velocity dispersion moments become linear if 
Table 4. Trend of the velocity dispersion element $\sigma_{R R}$ expressed in $\left[\mathrm{km} \mathrm{s}^{-1}\right]$.

\begin{tabular}{lcccccc}
\hline \hline$\sigma_{R R}(R, z)$ & $<-0.6$ & ]$-0.5,-0.3]$ & ]$-0.3,-0.1]$ & ]$-0.1,-0.1]$ & ] $0.1,0.3]$ & ] $0.3,0.5]$ \\
\hline 7.6 & & $36.6 \pm 22.9$ & & & & \\
7.8 & $45.6 \pm 18.5$ & $35.8 \pm 7.3$ & $32.7 \pm 20.4$ & & $31.2 \pm 25.0$ & $35.3 \pm 10.9$ \\
8.0 & $43.6 \pm 13.3$ & $34.8 \pm 4.0$ & $32.6 \pm 4.6$ & & $31.8 \pm 5.6$ & $34.0 \pm 5.5$ \\
8.2 & $41.3 \pm 11.1$ & $36.2 \pm 2.9$ & $32.1 \pm 1.5$ & $28.3 \pm 10.9$ & $32.4 \pm 1.8$ & $36.2 \pm 4.0$ \\
8.4 & $41.9 \pm 11.4$ & $35.4 \pm 2.1$ & $31.6 \pm 1.0$ & $27.4 \pm 1.1$ & $31.8 \pm 1.2$ & $34.7 \pm 3.8$ \\
8.6 & $41.7 \pm 13.8$ & $34.4 \pm 2.4$ & $31.0 \pm 1.2$ & $26.3 \pm 1.8$ & $31.9 \pm 1.9$ & $34.7 \pm 5.2$ \\
8.8 & $40.6 \pm 31.6$ & $34.0 \pm 4.0$ & $32.5 \pm 3.1$ & $24.8 \pm 31.6$ & $32.6 \pm 7.9$ & $33.9 \pm 9.2$ \\
9.0 & $40.9 \pm 35.3$ & $33.8 \pm 7.7$ & $31.8 \pm 14.5$ & & $31.9 \pm 37.7$ & $32.7 \pm 22.9$ \\
9.2 & & $32.3 \pm 17.6$ & & & & \\
\hline
\end{tabular}

Notes. The radial distance bins (rows in leftmost column) and vertical direction bins (column headers) are in [kpc]. Empty slots indicate regions where not enough stars were sampled.

Table 5. Trend of the velocity dispersion element $\sigma_{R \phi}$ expressed in $\left[\mathrm{km} \mathrm{s}^{-1}\right]$.

\begin{tabular}{lccccc}
\hline \hline$\sigma_{R \phi}(R, z)$ & ]$-0.5,-0.3]$ & ]$-0.3,-0.1]$ & ]$-0.1,0.1]$ & ] $0.1,0.3]$ & ] $0.3,0.5]$ \\
\hline 7.6 & $-10.6 \pm 22.9$ & & & & \\
7.8 & $-9.8 \pm 7.3$ & $-10.2 \pm 20.4$ & & $-10.1 \pm 24.3$ & $-9.7 \pm 10.8$ \\
8.0 & $-10.5 \pm 4.0$ & $-10.2 \pm 4.6$ & & $-10.0 \pm 5.6$ & $-9.9 \pm 5.5$ \\
8.2 & $-9.6 \pm 2.9$ & $-10.9 \pm 1.6$ & $-10.2 \pm 10.8$ & $-10.5 \pm 1.9$ & $-9.6 \pm 4.0$ \\
8.4 & $-10.3 \pm 2.2$ & $-8.9 \pm 1.0$ & $-9.8 \pm 1.2$ & $-9.3 \pm 1.2$ & $-9.1 \pm 3.9$ \\
8.6 & $-8.8 \pm 2.4$ & $-9.4 \pm 1.2$ & $-9.6 \pm 1.9$ & $-8.4 \pm 2.0$ & $-9.4 \pm 5.3$ \\
8.8 & $-8.8 \pm 4.0$ & $-9.7 \pm 3.2$ & $-9.7 \pm 31.6$ & $-10.0 \pm 8.0$ & $-8.4 \pm 9.2$ \\
9.0 & $-8.7 \pm 7.8$ & $-9.6 \pm 14.6$ & & $-9.8 \pm 37.8$ & $-8.3 \pm 22.9$ \\
9.2 & $-9.6 \pm 17.7$ & & & \\
\hline
\end{tabular}

Notes. The radial distance bins (rows in leftmost column) and vertical direction bins (column headers) are in [kpc]. Empty slots indicate regions where not enough stars were sampled.

Table 6. Trend of the velocity dispersion element $\sigma_{R z}$ expressed in $\left[\mathrm{km} \mathrm{s}^{-1}\right]$.

\begin{tabular}{lccccc}
\hline \hline$\sigma_{R z}(R, z)$ & ]$-0.5,-0.3]$ & ]$-0.3,-0.1]$ & ]$-0.1,0.1]$ & ] $0.1,0.3]$ & ] $0.3,0.5]$ \\
\hline 7.6 & $-22.7 \pm 22.9$ & & & $11.7 \pm 24.3$ & $17.8 \pm 10.8$ \\
7.8 & $-17.9 \pm 7.3$ & $-14.7 \pm 20.4$ & & $11.0 \pm 5.6$ & $16.2 \pm 5.5$ \\
8.0 & $-17.8 \pm 4.0$ & $-9.1 \pm 4.6$ & & $8.3 \pm 1.9$ & $12.9 \pm 4.0$ \\
8.2 & $-13.0 \pm 2.9$ & $-7.2 \pm 1.6$ & $-4.0 \pm 10.8$ & $-2.7 \pm 1.2$ & $9.8 \pm 3.9$ \\
8.4 & $-7.8 \pm 2.2$ & $-4.0 \pm 1.0$ & $-3.2 \pm 2.2$ & $-4.8 \pm 2.0$ & $2.4 \pm 5.3$ \\
8.6 & $-6.1 \pm 2.4$ & $-2.0 \pm 1.2$ & $-4.5 \pm 2.9$ & -4.8 \\
8.8 & $2.8 \pm 4.0$ & $5.8 \pm 3.2$ & $-4.8 \pm 31.6$ & $-9.9 \pm 8.0$ & $-4.7 \pm 9.2$ \\
9.0 & $8.1 \pm 7.8$ & $9.6 \pm 14.6$ & & $-12.5 \pm 37.8$ & $-6.4 \pm 22.9$ \\
9.2 & $15.4 \pm 17.7$ & & & & \\
\hline
\end{tabular}

Notes. The radial distance bins (rows in leftmost column) and vertical direction bins (column headers) are in [kpc]. Empty slots indicate regions where not enough stars were sampled.

and only if the mean velocities of the partial distribution have the same mean (i.e., in our case, we can correct the thin disk and thick disk only in the radial and vertical directions). In the azimuthal direction clearly this is not the case (as evident from Table 2), preventing us from using the result on the dispersion tensor for the thick disk of Paper I in order to correctly recover the value $v_{V, \odot}$ from Eq. (3) and Tables 4, 6 and 7.

2. While the total potential $\Phi_{\text {tot }}$ and the scale length, $h_{R}$, which enter in the density $\rho(R, z)$ distribution of the population, can be related thanks to the Poisson equation (see, e.g., Pasetto 2005) or the appendix of Vallenari et al. (2006), unfortunately the velocity dispersion errors presented below do not permit us to compute the terms $\frac{\partial \sigma_{R R}^{2}}{\partial R}$ and $\frac{\partial \sigma_{R z}^{2}}{\partial z}$.
3. The age-velocity dispersion relation implies that the spatial distribution and kinematics of the stars are strongly dependent on the age of the stars, i.e., the dispersion tensor has a strong dependence on the time $\sigma_{i j}=\sigma_{i j}(R, z, t)$ as well as on the scale height $h_{z}=h_{z}(t)$ (e.g., Wielen 1974, 1977). Hence, in Eq. (3) we can express the time dependence of $\overline{\boldsymbol{v}}_{\phi}$ by writing $\overline{\boldsymbol{v}}_{\phi}(R, z, t)=\overline{\boldsymbol{v}}_{\phi}\left(R, z, \sigma_{i j}(R, z, t)\right)$ or more briefly $\overline{\boldsymbol{v}}_{\phi}=\overline{\boldsymbol{v}}_{\phi}\left(\sigma_{i j}(R, z, t)\right)$ to show that if we assume $\frac{\partial \Phi_{\text {tot }}}{\partial t} \ll \frac{\partial \sigma_{i j}}{\partial t}$ then the dominant time dependence in $\overline{\boldsymbol{v}}_{\phi}$ is given by $\sigma_{i j}=\sigma_{i j}(R, z, t)$. In this case, it is convenient to extrapolate the value of the motion of the solar neighbourhood $\overline{\boldsymbol{v}}_{\phi}$ as $\overline{\boldsymbol{v}}_{\phi}=\lim _{t \rightarrow t_{\min }} \overline{\boldsymbol{v}}_{\phi}\left(\sigma_{i j}\left(\bar{R}_{\odot}, \bar{z}_{\odot}, t\right)\right)$ where $\left\{\bar{R}_{\odot}, \bar{z}_{\odot}\right\}$ then is the range $\{R, z\} \in] 8.2,8.4] \times]-0.1,0.1] \mathrm{kpc}$ of Table 2 and $t_{\min }$ is the time to consider a young population 
Table 7. Trend of the velocity dispersion element $\sigma_{\phi \phi}$ expressed in $\left[\mathrm{km} \mathrm{s}^{-1}\right]$.

\begin{tabular}{lccccc}
\hline \hline$\sigma_{\phi \phi}(R, z)$ & ]$-0.5,-0.3]$ & ]$-0.3,-0.1]$ & ]$-0.1,0.1]$ & ] $0.1,0.3]$ & ] $0.3,0.5]$ \\
\hline 7.6 & $28.3 \pm 22.9$ & & & & \\
7.8 & $27.2 \pm 7.3$ & $24.3 \pm 20.4$ & & $24.1 \pm 24.3$ & $30.3 \pm 10.8$ \\
8.0 & $26.8 \pm 4.0$ & $26.1 \pm 4.6$ & & $24.2 \pm 5.6$ & $27.0 \pm 5.5$ \\
8.2 & $24.8 \pm 2.9$ & $23.0 \pm 1.6$ & $20.3 \pm 10.8$ & $21.4 \pm 1.9$ & $26.5 \pm 4.0$ \\
8.4 & $23.8 \pm 2.2$ & $21.7 \pm 1.0$ & $20.8 \pm 1.2$ & $20.8 \pm 1.2$ & $23.7 \pm 3.9$ \\
8.6 & $24.8 \pm 2.4$ & $21.2 \pm 1.2$ & $19.6 \pm 1.9$ & $21.1 \pm 2.0$ & $24.2 \pm 5.3$ \\
8.8 & $24.4 \pm 4.0$ & $21.8 \pm 3.2$ & $18.1 \pm 31.6$ & $20.0 \pm 8.0$ & $23.0 \pm 9.2$ \\
9.0 & $24.7 \pm 7.8$ & $20.5 \pm 14.6$ & & $17.3 \pm 37.8$ & $22.9 \pm 22.9$ \\
9.2 & $24.2 \pm 17.7$ & & & & \\
\hline
\end{tabular}

Notes. The radial distance bins (rows in leftmost column) and vertical direction bins (column headers) are in [kpc]. Empty slots indicate regions where not enough stars were sampled.

Table 8. Trend of the velocity dispersion element $\sigma_{\phi z}$ expressed in $\left[\mathrm{km} \mathrm{s}^{-1}\right]$.

\begin{tabular}{lccccc}
\hline \hline$\sigma_{\phi z}(R, z)$ & ]$-0.5,-0.3]$ & ]$-0.3,-0.1]$ & ]$-0.1,0.1]$ & ] $0.1,0.3]$ & ] $0.3,0.5]$ \\
\hline 7.6 & $5.1 \pm 22.9$ & & & & \\
7.8 & $4.8 \pm 7.3$ & $3.3 \pm 20.4$ & & $5.2 \pm 24.3$ & $4.9 \pm 10.8$ \\
8.0 & $2.9 \pm 4.0$ & $2.1 \pm 4.6$ & & $4.5 \pm 5.6$ & $7.3 \pm 5.5$ \\
8.2 & $0.6 \pm 2.9$ & $4.3 \pm 1.6$ & $3.3 \pm 10.8$ & $3.4 \pm 1.9$ & $8.5 \pm 4.0$ \\
8.4 & $-1.6 \pm 2.2$ & $3.7 \pm 1.0$ & $3.0 \pm 1.2$ & $4.6 \pm 1.2$ & $9.6 \pm 3.9$ \\
8.6 & $-3.0 \pm 2.4$ & $5.8 \pm 1.2$ & $5.4 \pm 1.9$ & $6.0 \pm 2.0$ & $9.6 \pm 5.3$ \\
8.8 & $-3.6 \pm 4.0$ & $5.3 \pm 3.2$ & $3.1 \pm 31.6$ & $5.6 \pm 8.0$ & $10.8 \pm 9.2$ \\
9.0 & $-4.9 \pm 7.8$ & $4.8 \pm 14.6$ & & $6.5 \pm 37.8$ & $10.5 \pm 22.9$ \\
9.2 & $-4.7 \pm 17.7$ & & & & \\
\hline
\end{tabular}

Notes. The radial distance bins (rows in leftmost column) and vertical direction bins (column headers) are in [kpc]. Empty slots indicate regions where not enough stars were sampled.

in statistical collisionless equilibrium (e.g., Aumer \& Binney 2009). Nevertheless, by applying such a time binning (on the age of the stars) we should limit ourselves to the immediate solar neighbourhood, namely only the closest bin $\left\{\bar{R}_{\odot}, \bar{z}_{\odot}\right\}$ to deal with a suitable amount of stars per bin, which is not the goal of the present paper. Furthermore, if the origin of the age-velocity dispersion relation stands in the gravitational potential fluctuation caused by transient spiral structure (e.g., De Simone et al. 2004) the approximation $\frac{\partial \Phi_{\mathrm{tot}}}{\partial t} \ll \frac{\partial \sigma_{i j}}{\partial t}$ may fail and the previous argument for the use of Eq. (3) does not hold any more.

Nevertheless, we present these results in Table 2 to be complete. We postpone the study of the trend of $\bar{v}_{\phi}$ in the meridional plane to future works and different techniques (e.g., Moni Bidin et al. 2012, see also the conclusion in Sect. 4).

\subsubsection{Mean vertical motion}

Finally, the mean vertical motion is presented in Table 3. The value around the solar position is $7.2 \pm 1.3 \mathrm{~km} \mathrm{~s}^{-1}$ in excellent agreement with the standard adopted literature values (e.g., Binney \& Merrifield 1998). No apparent trend is evident within the errors, taking $\forall R$ and $\forall z$ into consideration.

\subsection{Velocity dispersion tensor}

The trend of the velocity ellipsoid along the radial and vertical direction of the Galaxy is scarcely known due to the solar location within the Galaxy disk $z_{\odot} \cong 0$ and the resulting dust absorption that affects the observations towards the Galactic centre.

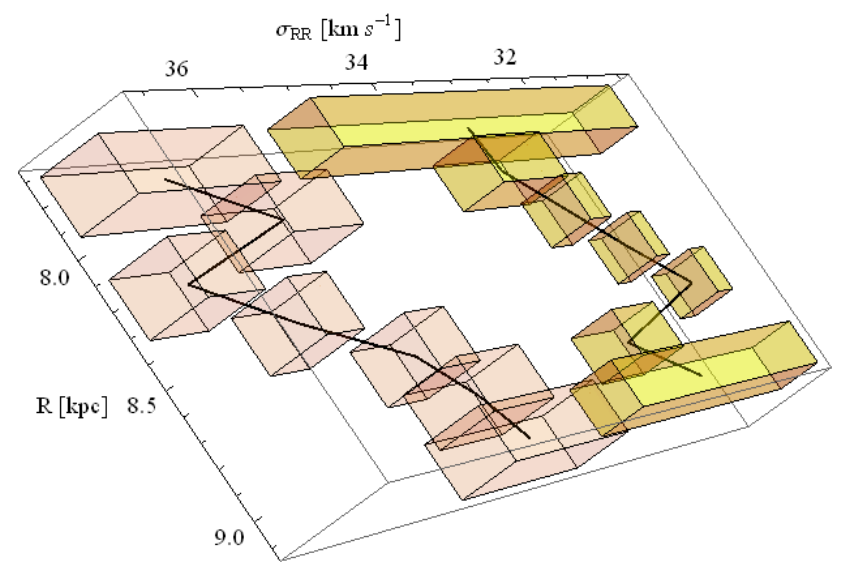

Fig. 3. An arbitrarily selected element and range from the $\sigma_{i j}$ tensor, here $\sigma_{R R}$ as an example of the three-dimensional error box that surrounds every point for two different vertical ranges (corresponding to the two upper panels of Fig. 4 for the same velocity dispersion tensor). Black lines connect the centres of the boxes.

The pioneering work of Lewis \& Freeman (1989) remains a solitary attempt to observationally address this difficult task and we also will limit ourselves only to the close solar neighbourhood by neglecting the use of giant stars in this work.

Nevertheless, the RAVE survey has the advantage of letting us work with an extended number of stars, and to permit us to sample the trend of the thin disk velocity ellipsoid in a radial range of $R \in[7.6,9.2] \mathrm{kpc}$ from the Galactic centre, an extension that is scarcely sampled in other works such as Lewis \& Freeman (1989). 


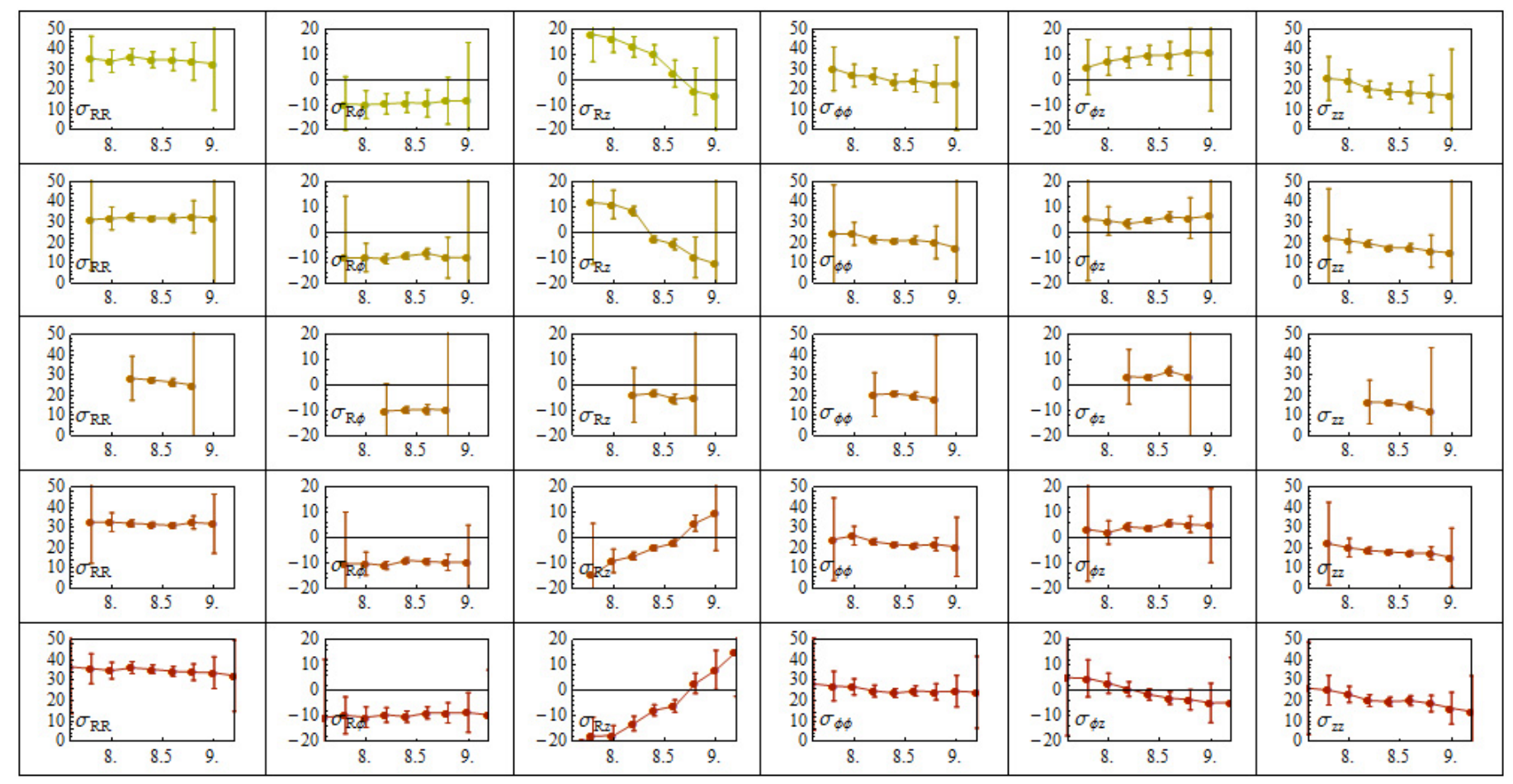

Fig. 4. Plot of all the velocity ellipsoid elements $\sigma_{i j}$ in the plane $(O, R, z)$. The axes are in $\left[\mathrm{km} \mathrm{s}^{-1}\right]$ for the velocity dispersion $(y$-axes) and in $[\mathrm{kpc}]$ for the Galactocentric distances ( $x$-axes). The axis labels are omitted in favour of a small indicator of the element we are considering in the lower left part of the plot. In order to visualize different $z$ distance ranges, we use a gradation of increasing red for the plot lines from the upper row of plot, $z \in] 0.3,0.5] \mathrm{kpc}$ down to the bottom row for the range $z \in]-0.5,0.3] \mathrm{kpc}$ : the first row of the upper 6 panels refers to $z \in] 0.3,0.5] \mathrm{kpc}$, the second to $z \in] 0.1,0.3] \mathrm{kpc}$, the central to the Galactic plane $z \in]-0.1,0.1] \mathrm{kpc}$. The fourth row includes panels for $z \in]-0.1,-0.3] \mathrm{kpc}$ and the fifth one to $z \in]-0.3,-0.5] \mathrm{kpc}$.

We consider now the analysis of the terms $\sigma_{R R}$ and $\sigma_{z z}$.

We start by looking at Fig. 3 where we show the trend of $\sigma_{R R}(R, z)$ with the Galactocentric distance $R$. The plot shows the trends of the data, but due to the overlapping of the error bars and the expected symmetry of the data above and below the plane caused by the proximity of the Sun to the ideal plane $z=0$, the plots with the error bars are generally crowded. We present an example of these plots only for illustrative purposes and only for the element $\sigma_{R R}$ in order to give to the reader a feeling of the behaviour of the error bars. For each data point, the errors are given by a triplet of numbers, $\left\{\Delta R, \Delta z, \Delta \sigma_{i j}(R, z)\right\}$, making a visualization hard to realize. Each point lies within a threedimensional error cube whose size is mainly a consequence of the error propagation formula (an element by element explicit derivation can be found in the Appendix of Fuchs et al. 2009) and it depends on the number of stars within the selected spatial range (which, in turn, is variable for each realization $P^{[j]}$ ).

In order to avoid realizing such a complicated threedimensional plot, in the figures we are presenting for a generic element, say $\sigma_{R R}(R, z)$ in Figs. 5-7, we are omitting the errors in the $z$ direction.

Moreover, as a general remark, we can observe how the error bars are typically smaller for the data sampled below the plane thanks to the better sampling of the RAVE data below the Galactic plane. Finally, due to the proximity of the sample to the solar location, we prefer to present a linear plot instead of a logarithmic one (see, e.g., Lewis \& Freeman 1989; van der Kruit $\&$ Searle 1981). The resulting trends for all the elements of the velocity dispersion tensor for the thin disk are shown in Fig. 4.

From Tables 4 and 9 we can compute directly the anisotropy parameter, which we define for the axisymmetric system as

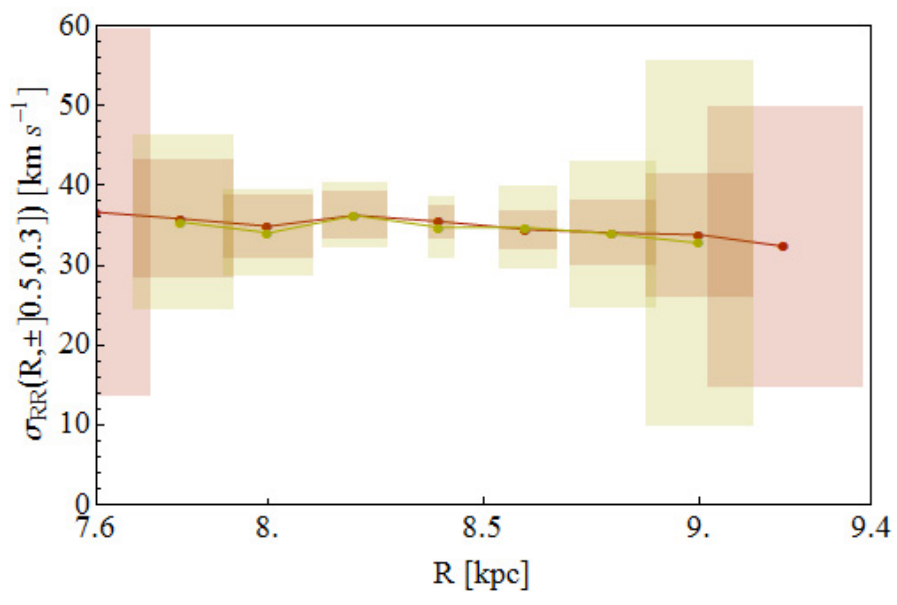

Fig. 5. Radial trend of the velocity dispersion element $\sigma_{R R}$ in the vertical interval $z \in] 0.3,0.5] \mathrm{kpc}$ (lighter colour) and $z \in]-0.5,-0.3] \mathrm{kpc}$ (darker colour). These vertical range intervals were plotted together in order to illustrate the symmetry above and below the disk plane (at $z=0)$.

$\beta(R, z) \equiv \frac{\sigma_{z z}^{2}}{\sigma_{R R}^{2}}$. This parameter is related to the first approach mentioned above. A plot of $\beta$ is presented in Figs. 8-10.

The trend for $\sigma_{z z}$ is presented in Table 9. Since the original works of van der Kruit \& Searle (1982; see also Antonuccio-Delogu 1991), it is commonly assumed that, for a self-gravitating thin disk at very small $z$, a single stellar population is vertically isothermal or only slightly non-isothermal (e.g., Amendt \& Cuddeford 1991; Bovy et al. 2012) and has a scale height nearly independent of the radius. Under this assumption 
Table 9. Trend of the velocity dispersion element $\sigma_{z z}$ expressed in $\left[\mathrm{km} \mathrm{s}^{-1}\right]$.

\begin{tabular}{lccccc}
\hline \hline$\sigma_{z z}(R, z)$ & ]$-0.5,-0.3]$ & ]$-0.3,-0.1]$ & ]$-0.1,0.1]$ & ] $0.1,0.3]$ & ] $0.3,0.5]$ \\
\hline 7.6 & $26.2 \pm 22.9$ & & & & \\
7.8 & $25.2 \pm 7.3$ & $22.1 \pm 20.4$ & & $22.1 \pm 24.3$ & $25.5 \pm 10.8$ \\
8.0 & $23.1 \pm 4.0$ & $20.1 \pm 4.6$ & & $20.8 \pm 5.6$ & $24.5 \pm 5.5$ \\
8.2 & $20.0 \pm 2.9$ & $18.7 \pm 2.6$ & $16.7 \pm 10.8$ & $19.4 \pm 1.9$ & $20.3 \pm 4.0$ \\
8.4 & $19.6 \pm 2.2$ & $18.0 \pm 2.0$ & $16.3 \pm 2.2$ & $17.3 \pm 1.2$ & $19.1 \pm 3.9$ \\
8.6 & $20.0 \pm 2.4$ & $17.5 \pm 2.2$ & $14.7 \pm 2.9$ & $17.3 \pm 2.0$ & $18.6 \pm 5.3$ \\
8.8 & $18.6 \pm 4.0$ & $17.4 \pm 3.2$ & $11.9 \pm 31.6$ & $15.7 \pm 8.0$ & $18.0 \pm 9.2$ \\
9.0 & $16.3 \pm 7.8$ & $15.2 \pm 14.6$ & & $14.9 \pm 37.8$ & $17.0 \pm 22.9$ \\
9.2 & $14.6 \pm 17.7$ & & & & \\
\hline
\end{tabular}

Notes. The radial distance bins (rows in leftmost column) and vertical direction bins (column headers) are in [kpc]. Empty slots indicate regions where not enough stars were sampled.

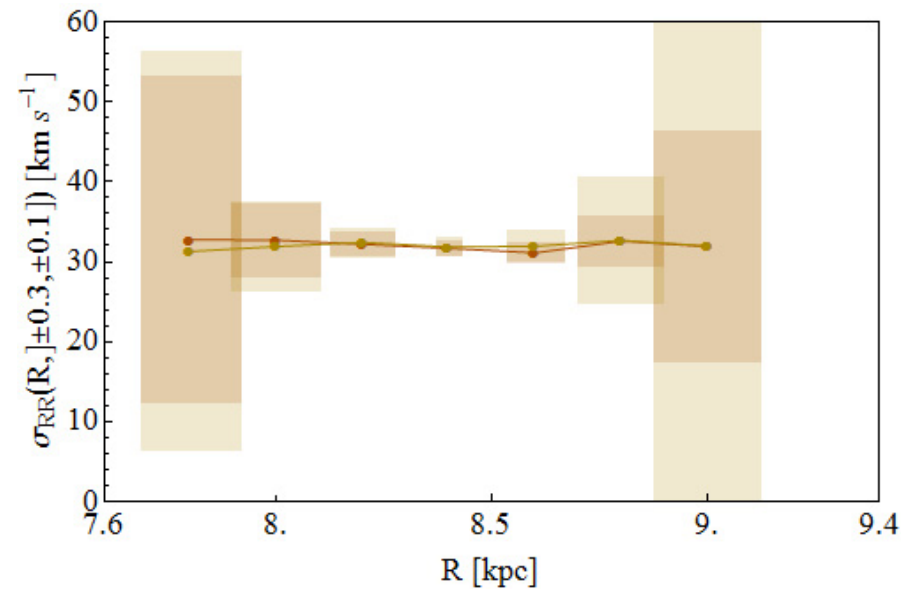

Fig. 6. Radial trend of the velocity dispersion element $\sigma_{R R}$ in the vertical interval $z \in] 0.1,0.3] \mathrm{kpc}$ (lighter colour) and $z \in]-0.3,-0.1] \mathrm{kpc}$ (darker colour). These vertical range intervals were plotted together in order to show the disk symmetry above and below the plane (at $z=0$ ).

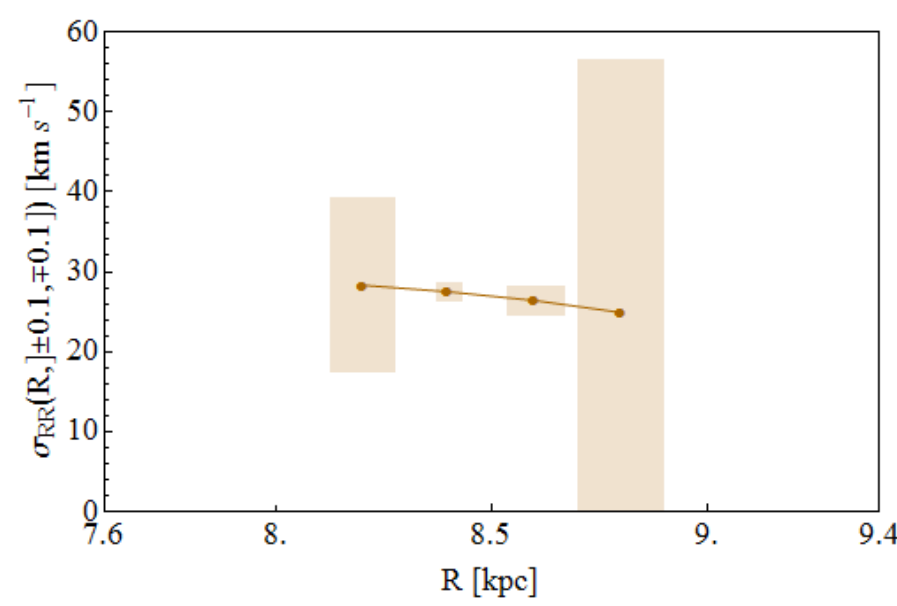

Fig. 7. Radial trend of the velocity dispersion element $\sigma_{R R}$ in the vertical interval $z \in]-0.1,0.1] \mathrm{kpc}$.

and by considering an exponential dependence of the radial light profile (Freeman 1970) and a constant mass-to-light ratio, the radial dependence of the vertical dispersion can be approximated as $\sigma_{z z}^{2}(R, 0)=\sigma_{z z}^{2}\left(R_{\odot}, 0\right) \mathrm{e}^{-\frac{R-R_{\odot}}{h_{R}}}$. A different approach is based on the local constancy of Toomre's stability parameter (Toomre 1964) which in epicyclical approximation leads to a

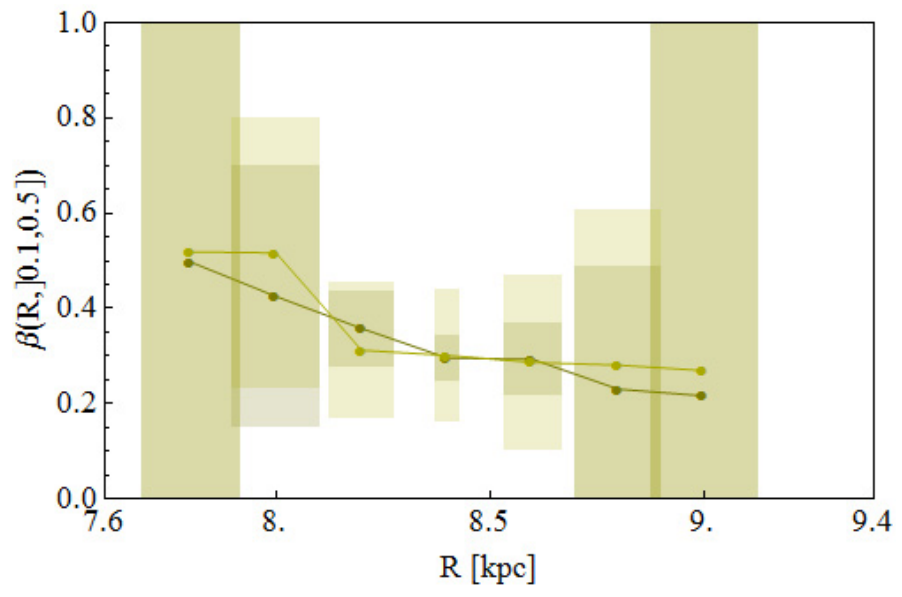

Fig. 8. Trend of the anisotropy parameter $\beta$ for the vertical distance range $z \in] 0.3,0.5] \mathrm{kpc}$ (lighter colour) and $z \in$ ]0.1, 0.3] kpc (darker colour).

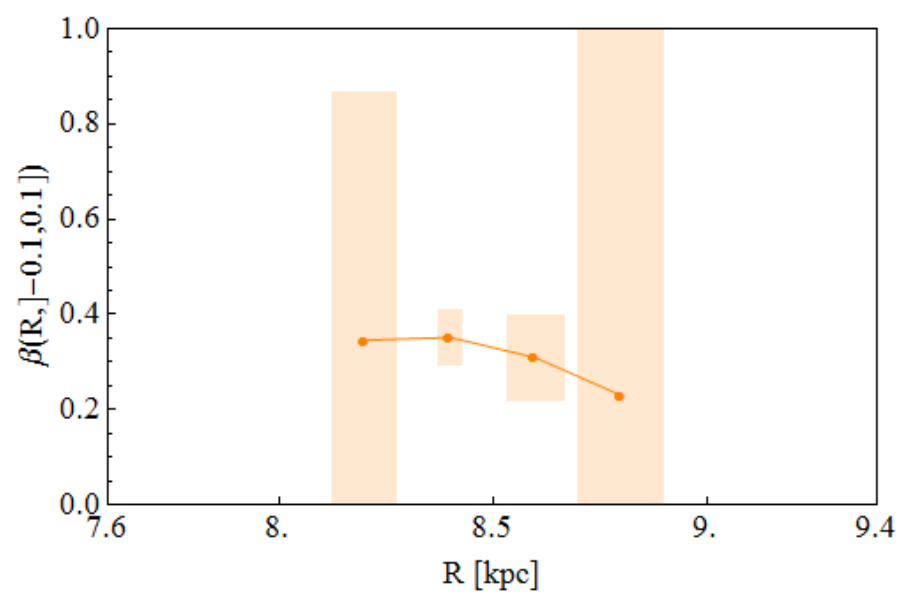

Fig. 9. Trend of the anisotropy parameter $\beta$ for $z \in]-0.1,0.1] \mathrm{kpc}$.

radial dependence of the form $\sigma_{R R}^{2}(R, 0)=\sigma_{R R}^{2}\left(R_{\odot}, 0\right) R^{2} \mathrm{e}^{-\frac{R-R_{\odot}}{h_{R}}}$ (e.g., Amendt \& Cuddeford 1991). Nevertheless, to first order for the solar radial distance $R-R_{\odot}$ both the relations result in a linear fit to the plot of Fig. 7 (see also Neese \& Yoss 1988).

A numerical solution for the velocity dispersion profile of the midplane at $z=0$ of the MW has been found by Cuddeford \& Amendt (1992). Their Eq. (22) is however dependent on the gradients of $\sigma_{z z}$ on the meridional plane or on the second derivative of the dispersion tensor. Nevertheless, our profiles 


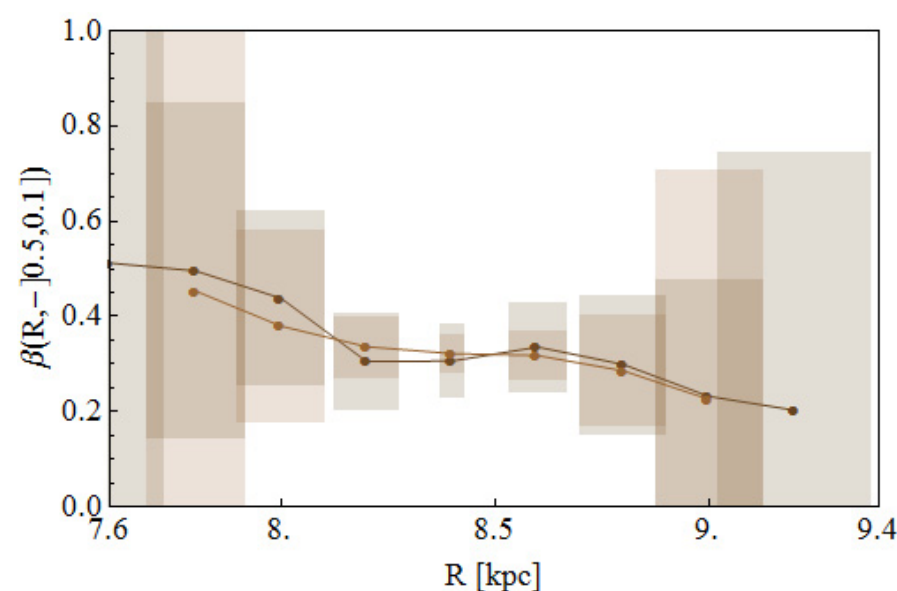

Fig. 10. Trend of the anisotropy parameter $\beta$ for the vertical distance range $z \in]-0.3,-0.1] \mathrm{kpc}$ (lighter colour) and $z \in]-0.5,-0.3] \mathrm{kpc}$ (darker colour).

can be used as a boundary condition for that type of analytical study.

In Fig. 11 we see the trend of the axis ratio of the velocity ellipsoid (e.g., Gilmore et al. 1989) along the meridional plane. In our approach we are dealing with a composite stellar population. Thus we do not necessarily expect to match the theoretical predictions that nevertheless remain based on several assumptions. The ratio $\frac{\sigma_{z z}(R, z)}{\sigma_{R R}(R, z)} \neq 1$ is shown in Fig. 11. In agreement with our initial hypotheses, once $f_{\text {thin }}=f_{\text {thin }}\left(E, L_{z}, I_{3}\right)$ is assumed for an axisymmetric and stationary thin disk with angular momentum $L_{z}$ and the energy $E$, then this trend has to be interpreted as observational evidence of the dependence of $f_{\text {thin }}$ on a third integral of motion $I_{3}$ and not on a deviation from the kurtosis values expected from the Schwarzschild distribution (see Paper I for further details). As can been seen, in the plane $\frac{\sigma_{z z}(R, 0)}{\sigma_{R R}(R, 0)} \in[0.4,0.5]$ and outside the plane we are close to constant within the error bars. Nevertheless, a small trend can be observed in Fig. 11 that can be interpreted as a suggestion of a small decrease of this ratio.

Finally in Table 9 the trend of $\sigma_{z z}=\sigma_{z z}(R, z)$ is reported. As is evident, the thin disk distribution of the mixture of main sequence stars seems to be remarkably isothermal (see, also Bovy et al. 2012). This is an apparent effect due to the mixture of the different populations acting to hide the velocity dispersion-age relation. The study of this relation is beyond the scope of this paper but can be easily achieved without a statistical procedure to disentangle the thin and thick disk component in order to consider a more relevant statistical example (or vice versa: age and chemistry can also be taken into consideration to improve the disentangling technique, see Sect. 4).

The coupling between vertical and radial direction is shown with the off diagonal term $\sigma_{R z}=\sigma_{R z}(R, z)$ in Table 6. This represents the tilt of the velocity ellipsoid in the radial direction in cylindrical coordinates. By symmetry we expect and we confirm that on the plane this tilt is approximately zero; $\sigma_{R z}(R, 0) \cong 0$. The tilt angle here has the remarkable meaning to represent a projection of the third integral of motion $I_{3}$ on the surface section of the meridional plane. Using $\theta=$ $\frac{1}{2}\left(\arctan \left(\frac{2 \sigma_{R z}^{2}}{\sigma_{R R}^{2}-\sigma_{z z}^{2}}\right)\right)$, we can obtain (from Tables 4, 6 and 9) a smoothly growing trend for the tilt as we get to higher $|z|$, even though the trend is not perfectly zero at our Sun's position $z=z_{\text {Sun }}$. Nevertheless, within the errors, the values are in agreement with the independent determination presented in

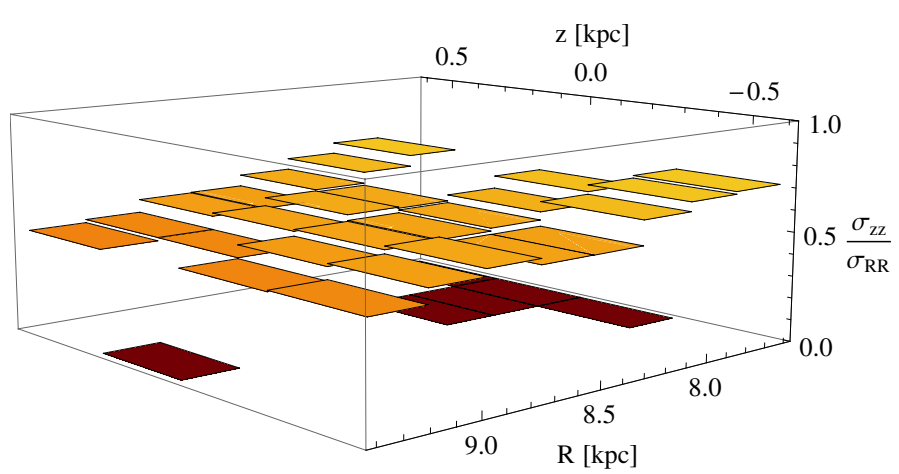

Fig. 11. Axis ratio $\sqrt{\beta}$ of the velocity ellipsoid in the meridional plane.

Vallenari et al. (2006) and the work of Amendt \& Cuddeford (1991) (or with the independent, compatible formulation by Bienaymé 2009).

Tables 5 and 7 report the values for $\sigma_{R \phi}=\sigma_{R \phi}(R, z)$ and $\sigma_{\phi \phi}=\sigma_{\phi \phi}(R, z)$ respectively. The first of these terms, $\sigma_{\varphi \varphi}=$ $\left(\bar{v}_{\varphi}-\bar{v}_{\varphi}\right)^{1 / 2}$ represents the second central moments of the azimuthal direction distribution. Despite the bias influencing the $\bar{v}_{\phi}$ determination, a smooth trend is still visible, which suggests an increasing velocity dispersion towards the Galactic centre as well as in the direction of increasing $|z|$ with remarkable symmetry above and below the plane. The second term, $\sigma_{R \phi}$ is null only in the case of axisymmetry, although in the literature we found considerable misuse of this assumption in the theoretical models. Nevertheless this assumption does not hold in the case of our Galaxy as seen in Table 5 and a greater effort should be made in order to relax the axisymmetry assumption in favour of a more permissive rotation symmetry about the $z$ axis for the thin disk DF, e.g., $f_{\text {thin }}(\phi)=f_{\text {thin }}(\phi+\pi)$. Actually, no analytical DF relaxing this approximation is known or applied. This term is strictly related to the phenomenon of the vertex deviation, i.e. the angular tilt of the velocity ellipsoid with the radial azimuthal direction by an angle $\psi$ so that $\tan (2 \psi)=\frac{2 \sigma_{R \phi}^{2}}{\sigma_{R R}^{2}-\sigma_{\phi \phi}^{2}}$. The problem is strictly related to the problem of the streaming motions. Its impact on the velocity ellipsoid in the solar neighbourhood has been emphasized by several authors (e.g., Soubiran et al. 2003; Dehnen 1998, 1999). Streaming motions can be recognized as local moving groups. Seabroke \& Gilmore (2007) show the presence of local moving groups complicates the age-velocity dispersion relation. As pointed out by Dehnen (1998) and De Simone et al. (2004), they affect the vertex deviation. Up to now, streaming motions have been studied only in the solar vicinity to get a local map of the velocity space (Bassino et al. 1986; Hilton \& Bash 1982; Mayor 1972; Woolley 1970; Dehnen 1998). Nevertheless, it is not possible to perform a check with the HipPaRcos data for the solar neighbourhood because the vast majority of the RAVE stars are outside of the range of the HIPPARcos parallaxes as well as the studies on the local vertex deviation. Finally the aforementioned problems with the first moment of the data distribution along the azimuthal direction is expected to influence the second central moments in this direction. In the left panel of Fig. 12 we show a graphical representation of the angular tilt of the velocity ellipsoid with the radial azimuthal direction. The RAVE data seem to support the idea that this tilt results as an effect of the streaming motions, showing a larger azimuthal tilt for stars closer to the plane.

Finally Table 8 shows the values derived for $\sigma_{\phi z}=\sigma_{\phi z}(R, z)$. They are considerably smaller than the previous terms or null 

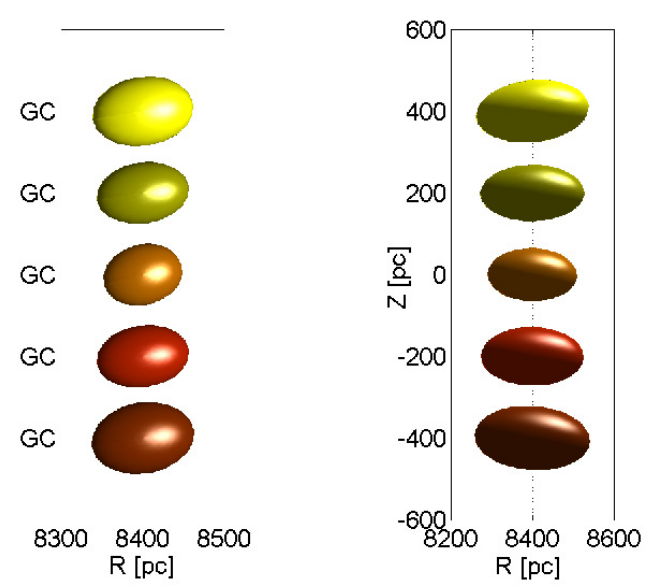

Fig. 12. Velocity ellipsoids in the solar cylinder as deduced from the velocity dispersions of Fig. 4 from two different points of view. On the left, we see the radial-azimuthal tilt of the velocity ellipsoids with respect to the direction of the Galactic centre (GC). The colours of the velocity ellipsoids refer to the height above or below the Galactic plane as indicated in the right panel (as well as in the vertical sequence of panels in Fig. 4). The right panel shows the vertical tilt of the velocity ellipsoids above and below the plane in the solar cylinder.

within the error bars. $\sigma_{\phi z}=0$ for a single stellar population tells us that the azimuthal and vertical velocities are approximately decoupled, or equivalently that the short axis of the velocity ellipsoid remains aligned with the cylindrical coordinate system (as expected at $z=0$ ). The same results seem to hold for the selected sample of dwarf stars in the thin disk.

\section{Conclusions}

The subject of this paper is the derivation the first two moments of the velocity distribution for the thin disk component based on stellar spectra for stars in the solar neighbourhood observed by RAVE. This was realized by disentangling the thin and thick disk; the thick disk has been the subject of our first paper (Pasetto et al. 2012c) and in the present paper we exclusively focus on the thin disk. Our main goal is the investigation of the solar motion relative to the LSR and the thin disk velocity dispersion trend. We derived two of the solar motion components as

$\left\{v_{U, \odot}, v_{W, \odot}\right\}=\{10.9 \pm 1.0,7.2 \pm 1.3\} \mathrm{km} \mathrm{s}^{-1}$.

All the results on the thin disk velocity ellipsoid can be summarized in a single figure, Fig. 13. Here we see the radial and vertical extensions that we have been able to map with the RAVE data as well as the ellipsoid axis ratios and their tilts. All the values come from Tables 4 to 9 .

There are several points that need a more extensive investigation. We just mention a few here:

- A methodology to obtain constraints on the disk potential and the radial velocity sample: a classical approach to this problem is based on a pre-assumed form for an analytical distribution function based on three isolating integrals of motion $f_{\text {thin }}=f_{\text {thin }}\left(I_{1}, I_{2}, I_{3}\right)$ from which by a projection procedure on the phase space, one can deduce analytically observable quantities directly linked to the potential. Unfortunately, these approaches, as the one adopted in Paper I, are subject to assumptions (axisymmetry, stationarity) that fail already in the solar neighbourhood: the most obvious case is perhaps the vertex deviation but see also recent

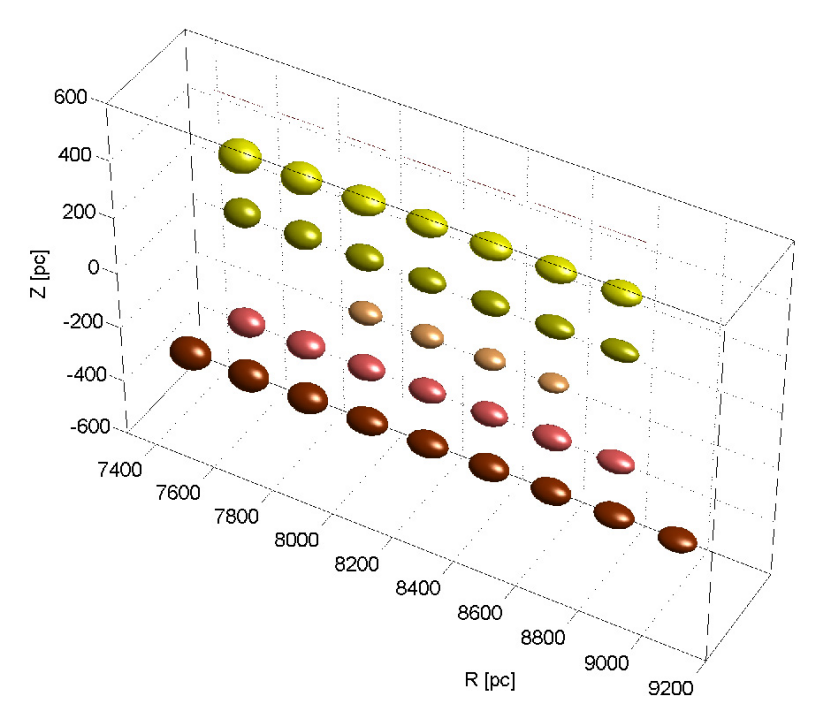

Fig. 13. Simple representation of the results on the velocity dispersion trend. The velocity dispersion tensor in each spatial point $(R, z)$ can be represented with an ellipsoidal surface defined by $\sigma_{i j}^{-2} v_{i} v_{j}=1$ (by summing over repeated indices). Here we plotted the velocity ellipsoids with their axes tabulated in the previous Tables 4, 9, 7 and their tilt listed in Tables 5, 6 and 8 . The colour gradient ranges from light colours above the plane to darker colours below the plane.

work by Luck et al. (2011) and Balser et al. (2011). Hence it would be useful to have an analytical model able to relax these assumptions in order to fully exploit the possibilities of the RAVE survey.

- The relation between stellar evolution, chemistry and the kinematics (the velocity dispersion) has been neglected in this paper. The reason is that in order to obtain a reasonable amount of stars and a reasonable precision in the dispersion tensor determination by mapping of the meridional plane, we should work with a limited subsample of stars closer to us. Nevertheless, it is in principle possible to further narrow down the thin disk component with selection criteria based on chemical arguments. For example, Neves et al. (2009) determined the trend of abundance ratios as a function of $[\mathrm{Fe} / \mathrm{H}]$ from 451 high-resolution spectra of $\mathrm{F}$, $\mathrm{G}$, and $\mathrm{K}$ main-sequence stars in the solar neighbourhood confirming a dichotomy in $[\alpha / \mathrm{Fe}]$ found already by Reddy et al. (e.g., 2006) or Fuhrmann (2004). Navarro et al. (2011) investigated the possibility of disentangling thin and thick disk stars using a combination of $[\mathrm{Fe} / \mathrm{H}],[\alpha / \mathrm{Fe}]$ and the heavy element $\mathrm{Eu}$ for a sample of 306 stars. Models mixing kinematics and chemistry are currently in development (e.g., Schönrich \& Binney 2009). Their results require further investigations with respect to the role of the radial migration of stars (e.g., Brunetti et al. 2011; Bensby et al. 2011; Bird et al. 2012; Grand et al. 2012) or gas (e.g., Spitoni \& Matteucci 2011) and the presence of metallicity gradients. Nevertheless, the RAVE catalogue is surely suitable for this kind of investigation since more and more data are being obtained and an extension of this disentangling technique to include giant stars and chemical constraints is worthy of investigation.

- Although the average values for $\bar{v}_{R}, \bar{v}_{\phi}$ and $\bar{v}_{z}$ present a smooth trend, we are not yet able to compute their derivatives $\partial_{R} \bar{v}_{i}$ or $\partial_{z} \bar{v}_{i}$, which remain targets of primary importance of forthcoming surveys such as Gaia (e.g., Robin et al. 2012). These elements are of fundamental importance in order to 
investigate the shape of the thin disk distribution function in order to clarify the importance of the third integral of motion over the deviation from the expected kurtosis value for a Schwarschild DF.

For the time being, however, the direct comparison of this observational work can be done only with other observational works. Finally a few cautionary remarks on the limit of our results. As already commented in the introduction, the extrapolation of the solar neighbourhood kinematic description to the whole Galactic disk relies on the validity of the Jeans theorem in the integral description of the thin disk distribution function. This is subject to the validity of the stationary condition that holds in a broad sense for the group of stars sampled by the RAVE survey. Nevertheless, we recall that several processes may cause small violations of this assumption in the solar neighbourhood, for instance:

- Spiral structures and the bar redistribute angular momentum across the disk and heat it up (e.g., Carlberg \& Sellwood 1985 ) by causing small deviations from the steady-state situation and potentially are indeed a main source influencing the second order moment of the $f_{\text {thin }}$ here studied. Minchev \& Famaey (2010) show an example of mixing by combined effects of bar and spirals even if the effect that they registered seems to be short-lived.

- The Galaxy accretes angular momentum from the remnants of external dwarf galaxies that are merging with it and the accretion is expected to lead to significant reorientation of the spin axis (e.g., Binney \& May 1986; Sellwood \& Binney 2002) by potentially influencing the trend of the first moment of $f_{\text {thin }}$.

Other processes that are not naturally considered in the stationary description include, e.g., the influence of the Outer Lindblad Resonance of the Galactic bar in the solar neighborhood (e.g., Dehnen 1999) or the constant stripping of small stellar streams from satellite galaxies (e.g., Helmi \& White 1999). These processes are expected to influence the data in the RAVE catalogue when also the giant stars are taken into consideration.

Acknowledgements. S.P. wants to thank B. Fuchs, A. Just and J. Binney for comments on the technique and results of the paper and I. Minchev for careful reading of the manuscript. Numerical computations have been partially performed with supercomputers at the John von Neumann - Institut für Computing (NIC) - Germany (NIC-project number 2979). We acknowledge partial funding from Sonderforschungsbereich SFB 881 "The Milky Way System" (subprojects A5 and A6) of the German Research Foundation (DFG). Funding for RAVE has been provided by: the Australian Astronomical Observatory; the Leibniz-Institut fuer Astrophysik Potsdam (AIP); the Australian National University; the Australian Research Council; the French National Research Agency; the German Research Foundation (SPP 1177 and SFB 881); the European Research Council (ERC-StG 240271 Galactica); the Istituto Nazionale di Astrofisica at Padova; The Johns Hopkins University; the National Science Foundation of the USA (AST-0908326); the W. M. Keck foundation; the Macquarie University; The Netherlands Research School for Astronomy; the Natural Sciences and Engineering Research Council of Canada; the Slovenian Research Agency; the Swiss National Science Foundation; the Science \& Technology Facilities Council of the UK; Opticon; Strasbourg Observatory; and the Universities of Groningen, Heidelberg and Sydney. The RAVE web site is at http://www . rave-survey.org.

\section{References}

Amendt, P., \& Cuddeford, P. 1991, ApJ, 368, 79

Antonuccio-Delogu, V. 1991, A\&A, 247, 45

Aumer, M., \& Binney, J. J. 2009, MNRAS, 397, 1286

Bahcall, J. N., \& Soneira, R. M. 1980, ApJS, 44, 73

Balser, D. S., Rood, R. T., Bania, T. M., \& Anderson, L. D. 2011, ApJ, 738, 27
Bassino, L. P., Dessaunet, V. H., \& Muzzio, J. C. 1986, Rev. Mex. Astron. Astrofis., 13, 9

Bensby, T., Alves-Brito, A., Oey, M. S., Yong, D., \& Meléndez, J. 2011, ApJ, 735, L46

Bertelli, G., Vallenari, A., Pasetto, S., \& Chiosi, C. 2003, in GAIA Spectroscopy: Science and Technology, ed. U. Munari, ASP Conf. Ser., 298, 153

Bertin, G. 2000, Dynamics of Galaxies (Cambridge University Press)

Bienaymé, O. 2009, A\&A, 500, 781

Bienayme, O., Robin, A. C., \& Creze, M. 1987, A\&A, 180, 94

Binney, J., \& May, A. 1986, MNRAS, 218, 743

Binney, J., \& Merrifield, M. 1998, Galactic astronomy (Princeton University Press)

Binney, J., \& Tremaine, S. 1987, Galactic dynamics (Princeton University Press)

Bird, J. C., Kazantzidis, S., \& Weinberg, D. H. 2012, MNRAS, 420, 913

Boeche, C., Siebert, A., Williams, M., et al. 2011, AJ, 142, 193

Bovy, J., Rix, H.-W., Hogg, D. W., et al. 2012, ApJ, 755, 115

Brunetti, M., Chiappini, C., \& Pfenniger, D. 2011, A\&A, 534, A75

Carlberg, R. G., \& Sellwood, J. A. 1985, ApJ, 292, 79

Carroll, B. W., \& Ostlie, D. A. 2006, An introduction to modern astrophysics and cosmology (Pearson 1986)

Casetti-Dinescu, D. I., Girard, T. M., Korchagin, V. I., \& van Altena, W. F. 2011, ApJ, 728, 7

Coşkunoğlu, B., Ak, S., Bilir, S., et al. 2011, MNRAS, 412, 1237

Coşkunoğlu, B., Ak, S., Bilir, S., et al. 2012, MNRAS, 419, 2844

Combes, F., Boisse, P., Mazure, A., Blanchard, A., \& Seymour, M. 1995, Galaxies and Cosmology (Springer-Verlag)

Cubarsi, R., \& Alcobé, S. 2004, A\&A, 427, 131

Cuddeford, P., \& Amendt, P. 1992, MNRAS, 256, 166

De Simone, R., Wu, X., \& Tremaine, S. 2004, MNRAS, 350, 627

Dehnen, W. 1998, AJ, 115, 2384

Dehnen, W. 1999, ApJ, 524, L35

Dehnen, W. 2009, MNRAS, 395, 1079

Eggen, O. J., Lynden-Bell, D., \& Sandage, A. R. 1962, ApJ, 136, 748

Freeman, K. C. 1970, ApJ, 160, 811

Fuchs, B., Dettbarn, C., Rix, H.-W., et al. 2009, AJ, 137, 4149

Fuhrmann, K. 2004, Astron. Nachr., 325, 3

Fulbright, J. P., Wyse, R. F. G., Ruchti, G. R., et al. 2010, ApJ, 724, L104

Fux, R. 1997, A\&A, 327, 983

Gilmore, G., \& Reid, N. 1983, MNRAS, 202, 1025

Gilmore, G., Wyse, R. F. G., \& Kuijken, K. 1989, ARA\&A, 27, 555

Grand, R. J. J., Kawata, D., \& Cropper, M. 2012, MNRAS, 421, 1529

Hahn, C. H., Sellwood, J. A., \& Pryor, C. 2011, MNRAS, 418, 2459

Helmi, A., \& White, S. D. M. 1999, MNRAS, 307, 495

Hilton, J. L., \& Bash, F. 1982, ApJ, 255, 217

Johnson, D. R. H., \& Soderblom, D. R. 1987, AJ, 93, 864

Karataş, Y., \& Klement, R. J. 2012, New A, 17, 22

Kiss, L. L., Moór, A., Szalai, T., et al. 2011, MNRAS, 411, 117

Klement, R., Fuchs, B., \& Rix, H.-W. 2008, ApJ, 685, 261

Lewis, J. R., \& Freeman, K. C. 1989, AJ, 97, 139

Luck, R. E., Andrievsky, S. M., Kovtyukh, V. V., Gieren, W., \& Graczyk, D. 2011, AJ, 142, 51

May, A., \& Binney, J. 1986, MNRAS, 221, 857

Mayor, M. 1972, A\&A, 18, 97

Mendez, R. A., Rich, R. M., van Altena, W. F., et al. 1996, in The Galactic Center, ed. R. Gredel, ASP Conf. Ser., 102, 345

Minchev, I., \& Famaey, B. 2010, ApJ, 722, 112

Moni Bidin, C., Carraro, G., \& Méndez, R. A. 2012, ApJ, 747, 101

Navarro, J. F., Abadi, M. G., Venn, K. A., Freeman, K. C., \& Anguiano, B. 2011, MNRAS, 412, 1203

Neese, C. L., \& Yoss, K. M. 1988, AJ, 95, 463

Neves, V., Santos, N. C., Sousa, S. G., Correia, A. C. M., \& Israelian, G. 2009, A\&A, 497, 563

Partridge, R. B., \& Peebles, P. J. E. 1967, ApJ, 147, 868

Pasetto, S. 2005, Ph.D. Thesis (University of Padua)

Pasetto, S., Chiosi, C., \& Carraro, G. 2003, A\&A, 405, 931

Pasetto, S., Grebel, E. K., Zwitter, T., et al. 2012, A\&A, 547, A70 (Paper I)

Piskunov, A. E., Kharchenko, N. V., Röser, S., Schilbach, E., \& Scholz, R. 2006, A\&A, 445, 545

Quillen, A. C., Dougherty, J., Bagley, M. B., Minchev, I., \& Comparetta, J. 2011, MNRAS, 417, 762

Reddy, B. E., Lambert, D. L., \& Allende Prieto, C. 2006, MNRAS, 367, 1329

Robin, A. C., Luri, X., Reylé, C., et al. 2012, A\&A, 543, A100

Ruchti, G. R., Fulbright, J. P., Wyse, R. F. G., et al. 2010, ApJ, 721, L92

Ruchti, G. R., Fulbright, J. P., Wyse, R. F. G., et al. 2011, ApJ, 743, 107

Saslaw, W. C. 1985, Gravitational physics of stellar and galactic systems (Cambridge University Press)

Schönrich, R. 2012, MNRAS, in press [arXiv: 1207.3079]

Schönrich, R., \& Binney, J. 2009, MNRAS, 396, 203 


\section{S. Pasetto et al.: Thin disk with RAVE}

Schönrich, R., Binney, J., \& Dehnen, W. 2010, MNRAS, 403, 1829 Seabroke, G. M., \& Gilmore, G. 2007, MNRAS, 380, 1348

Seabroke, G. M., Gilmore, G., Siebert, A., et al. 2008, MNRAS, 384, 11 Searle, L., \& Zinn, R. 1978, ApJ, 225, 357

Sellwood, J. A., \& Binney, J. J. 2002, MNRAS, 336, 785

Siebert, A., Bienaymé, O., Binney, J., et al. 2008, MNRAS, 391, 793

Siebert, A., Famaey, B., Minchev, I., et al. 2011, MNRAS, 412, 2026

Skrutskie, M. F., Cutri, R. M., Stiening, R., et al. 2006, AJ, 131, 1163

Soubiran, C., Bienaymé, O., \& Siebert, A. 2003, A\&A, 398, 141

Spitoni, E., \& Matteucci, F. 2011, A\&A, 531, A72

Steinmetz, M., Zwitter, T., Siebert, A., et al. 2006, AJ, 132, 1645

Toomre, A. 1964, ApJ, 139, 1217

Trumpler, R. J., \& Weaver, H. F. 1953, Statistical astronomy (Dover Publications)
Vallenari, A., Pasetto, S., Bertelli, G., et al. 2006, A\&A, 451, 125

van der Kruit, P. C., \& Freeman, K. C. 2011, ARA\&A, 49, 301

van der Kruit, P. C., \& Searle, L. 1981, A\&A, 95, 105

van der Kruit, P. C., \& Searle, L. 1982, A\&A, 110, 61

Veltz, L., Bienaymé, O., Freeman, K. C., et al. 2008, A\&A, 480, 753

Vio, R., Fasano, G., Lazzarin, M., \& Lessi, O. 1994, A\&A, 289, 640

Wielen, R. 1974, Highlights of Astronomy, 3, 395

Wielen, R. 1977, A\&A, 60, 263

Williams, M. E. K., Steinmetz, M., Sharma, S., et al. 2011, ApJ, 728, 102

Wilson, M. L., Helmi, A., Morrison, H. L., et al. 2011, MNRAS, 413, 2235 Woolley, R. 1970, in The Spiral Structure of our Galaxy, eds. W. Becker, \& G. I. Kontopoulos, IAU Symp., 38, 423

Zwitter, T., Siebert, A., Munari, U., et al. 2008, AJ, 136, 421

Zwitter, T., Matijevič, G., Breddels, M. A., et al. 2010, A\&A, 522, A54 